\title{
Solar active regions: SOHO/CDS and TRACE observations of quiescent coronal loops
}

\author{
G. Del Zanna and H. E. Mason \\ Department of Applied Mathematics and Theoretical Physics, University of Cambridge, UK \\ Received 12 February 2003 / Accepted 20 May 2003
}

\begin{abstract}
This study focuses on the diagnostic capabilities of the TRACE and SOHO-CDS instruments to describe the physical characteristics of quiescent solar active regions. In particular we concentrate on the $1 \mathrm{MK}$ loops, best seen in the TRACE EUV $173 \AA$ A passband. These are high-lying structures which can remain stable over timescales of hours to days. We present simultaneous observations of off-limb active region 1 MK loops performed with TRACE (173 $⿱$ and $195 \AA$ bands) and the SOHO-CDS. The CDS spectral capabilities complement the TRACE observations well and allow an in-depth study of the plasma properties. Some important new results are presented here. In terms of cross-field characteristics, we find that these loops are isothermal and much cooler $\left(T \simeq 0.7-1.1 \times 10^{6} \mathrm{~K}\right)$ than the surrounding diffuse high-lying active region emission $\left(T \simeq 2 \times 10^{6} \mathrm{~K}\right)$. We show that the diffuse background-foreground constitutes the bulk of the high-lying quiescent active region emission. We explain some of the discrepancies found by various authors (based on SOHO and TRACE observations) with an overestimation of loop temperatures and densities. This was caused by inaccurate or neglected background subtraction in the data analysis. We studied the leg of a $1 \mathrm{MK}$ loop and found that along its length temperatures and densities have steeper profiles compared to those derived from previous studies based on TRACE observations. The observations are consistent with an element abundance FIP effect of about 4, filling factors close to unity, and a constant density across the loop. We find a remarkable consistency between the CDS and TRACE observations, although we also find severe limitations to the use of TRACE diagnostic techniques.
\end{abstract}

Key words. Sun: corona - techniques: spectroscopic - Sun: transition region - Sun: abundances

\section{Introduction}

The Transition Region and Coronal Explorer (TRACE) instrument is exceptional in terms of high spatial resolution $\left(0.5^{\prime \prime}\right)$, high temporal resolution (of the order of $30 \mathrm{~s}$ or less) and also a relatively large field of view. TRACE has provided some beautiful EUV images and movies ${ }^{1}$, which have changed our view of the solar corona, in particular of active regions (ARs), with their ubiquitous loop structures. Loops have been studied for a long time with many instruments flown in space, including those on-board Skylab and the Solar and Heliospheric Observatory (SOHO). The literature is extensive (see, e.g., Orrall 1981; Bray 1991, and references therein for the status of the knowledge before SOHO and TRACE were launched). Briefly, loops have a wide range of sizes, temperatures and lifetimes. The spatial and temporal relationship between different loops remains elusive. Active regions are often characterised by many events that complicate the picture, such as magnetic flux emergence, continuous small scale flaring (see e.g. Del Zanna 2002a), occasional large flares, post-flare loops, and filament eruptions (see, e.g., Gibson et al. 2002; Pike \& Mason 2002).

\footnotetext{
Send offprint requests to: G. Del Zanna, e-mail: G.Del-Zanna@damtp.cam.ac.uk

${ }^{1}$ see, e.g. the CDROM in Sol. Phys. vol. 187, 1999, and the TRACE WWW page http://vestige.lmsal.com/TRACE/
}

Even without direct evidence of flares, many active regions are very dynamic. For example, they produce "cool" $(T \sim$ $10^{5} \mathrm{~K}$ ) transition region loops with large flows (see, e.g. Brekke et al. 1997). Often "hot" loops are also present but not necessarily co-spatial (see, e.g. Fludra et al. 1997; Kjeldseth-Moe \& Brekke 1998). Hot and cool loops can change their appearance over timescales of minutes.

Nonetheless, a large number of active regions experience a relatively "quiescent" stage, in which their morphology does not appreciably change over long periods of time, hours or even days. In this paper, we focus on the characteristics of the "typical" quiescent coronal loops. These loops are seen in upper transition-region or coronal lines (emitted at $T=0.7-1 \mathrm{MK}$ ) and represent the bulk of quiescent AR loops observed in the TRACE $173 \AA$ and SOHO/EIT (EUV Imaging Telescope) $171 \AA ̊$ A bandpassess. We shall hereafter call them "1 MK loops". These loops are long lived (hours to days) and are very extended. They are normally anchored in strong unipolar regions and connect either different active regions or the two polarities across the neutral line (see, e.g., Schrijver et al. 1999).

Quiescent active region loops are the simplest structures which need to be understood, before attempting to explain more complex and dynamic features. However, the current observational knowledge of even these loops is rather limited. The presence of $1 \mathrm{MK}$ loops in active regions has been known for 
more than three decades from Skylab and other XUV observations (see, e.g., Cheng 1980). However, most authors have either concentrated on the cool loops seen in transition region lines (formed in the range 50000-500000 K) or on the hot loops (mostly post-flare), observed in lines formed at temperatures of at least $1.5-3 \mathrm{MK}$. Indeed, there are only a few papers with detailed spectroscopic studies of coronal loops. Before SOHO, notable exceptions are the studies of Gabriel \& Jordan (1975), Dere (1982), Raymond \& Foukal (1982) and Doyle et al. (1985). However, in most of these cases the attention was drawn to the cool emission, where diagnostics were available. These early observations had various limitations in terms of temporal, spectral and spatial resolution.

Recently, several authors have used TRACE data to study the characteristics of quiescent coronal loops. The studies include those by Lenz et al. (1999), Aschwanden et al. (2000), Chae et al. (2002), and Testa et al. (2002). Other works (Aschwanden et al. 1999; Neupert et al. 1998) were based on SOHO-EIT observations. Lenz et al. (1999) analysed 4 TRACE loops, assuming that they are isothermal along the line of sight. They found nearly flat emission measure and temperature profiles along the length of the loops. The temperatures were all the same, $T \simeq 1.3 \mathrm{MK}$. These results are in stark contrast with the predictions of hydrostatic models such as that one of Serio et al. (1981), which predict a steep temperature decrease toward the loop footpoints, and much lower emission measures than those observed. Aschwanden et al. (2000) analysed a large number of TRACE loops, deriving density and temperature profiles. The maximum temperature for each loop was obtained assuming that each TRACE loop is composed of many threads of different temperatures, and using an hydrostatic model. Aschwanden et al. find slightly lower maximum temperatures compared to those derived by Lenz et al. (in the range 1.1-1.4 MK for the same loops) but overall confirm their results, with temperatures decreasing only by about $10 \%$ toward the footpoints for most loops.

Overall, the published results based on TRACE and EIT show some contradictions, which at first sight could be attributed to the diagnostic techniques and the uncertainties associated to the nature of the TRACE and EIT instruments which produce images in spectral bands of a few $\AA$. The best way to confirm results based on imaging instruments is to analyse simultaneous spectral data. The Coronal Diagnostic Spectrometer (CDS) on board SOHO is exceptional in terms of its diagnostic capabilities, for example to determine emission measures, electron temperatures and densities. The main drawback with CDS is its relatively low spatial resolution (of the order of $6-7^{\prime \prime}$ ) and the length of time required to scan the field of view.

There are several publications based on CDS observations of AR loops (e.g., Mason et al. 1999), but only a few papers have dealt with detailed studies of a coronal loop. The most detailed work is that of Schmelz et al. (2001). Their results apparently conflict with the TRACE results, primarily because the loop analysed by Schmelz et al. did not appear to be isothermal, either along the line of sight, or along its axis. This contradiction has led to intense debate and several other recent papers on the subject (see, e.g., Reale \& Peres 2000; Aschwanden 2002; Martens et al. 2002; Schmelz et al. 2002).

We have re-analysed the loop described in Schmelz et al. (2002), and one of the loops analysed by Lenz et al. (1999) and Aschwanden et al. (2000). We contend that the fact that different authors have obtained very different results has two main causes. First, the background emission has been neglected or treated improperly in the data analysis, leading to an overestimation of loop densities and temperatures. These loops were observed off-limb, where contamination by foreground and background emission is high. This contamination (called "diffuse background" in this paper), constitutes the bulk of the high-lying active region emission. Second, the loops observed by TRACE and CDS were intrinsically different types of loops.

The main aim of this paper is to use simultaneous multifilter TRACE and CDS observations to define the true nature of quiescent active region loops. This has been achieved by:

a) critically assessing the diagnostic methods and capabilities of the TRACE and CDS instruments for the study of these 1 MK loops;

b) investigating the importance of subtracting the background emission, both for spectral and imaging data, and how it affects measurements;

c) comparing results obtained from CDS and TRACE.

In Sect. 2 we present a short description of the CDS and TRACE instruments and their diagnostic capabilities. In Sect. 3 we present the observations and results based on the direct CDS-TRACE comparison and on the CDS diagnostics. In Sect. 4 we discuss the TRACE diagnostics, while in Sect. 5 we draw the conclusions.

\section{The TRACE and CDS instruments}

The TRACE instrument observes the solar corona in three EUV filters, centred around 173, 195, $284 \AA$. TRACE is described in Handy et al. (1999) and in the TRACE User Guide written by B. Bentley. The TRACE EUV filters are similar to those of the EIT instrument (Delaboudiniere et al.1995), centred around 171,195 , and $284 \AA$.

For both TRACE and EIT, the diagnostics (temperatures and emission measures) are obtained with the filter ratio technique (see Sect. 4 for details). It is common to assume that most of the emission in the three coronal bands is due to Fe IX-X, Fe XII and Fe XV respectively, and to assume that the plasma is isothermal along the line of sight. For both EIT and TRACE, the first two coronal filters are the most widely used, since the $284 \AA$ filter has a much lower sensitivity.

There are various problems with the filter ratio technique. One is that the coronal bands are not isothermal, in the sense that lines that are formed over a wide range of temperatures contribute to the signal in each band. For the EIT, this has been discussed in Del Zanna et al. (2003) with a direct comparison between CDS spectra and EIT images. The TRACE case is discussed in Sect. 4 below. The various problems have the effect that temperatures derived from the filter ratios are not uniquely determined, and can be very different from the temperatures derived directly from the spectra. 
The CDS observes many emission lines from a large number of ions, covering a wide range of temperatures. It is therefore the perfect instrument to complement imaging observations, because temperatures can be accurately determined. CDS consists of two instruments (described in Harrison et al. 1995): a Normal Incidence Spectrometer (NIS) and a Grazing Incidence Spectrometer (GIS). Here we only deal with NIS observations. The NIS detectors observe two spectral ranges simultaneously (NIS 1: 308-379 ̊̊ and NIS 2: 513-633 ̊). CDS can provide monochromatic images with a $4^{\prime} \times 4^{\prime}$ field of view by rastering of a long slit (from West to East).

Different spectroscopic techniques can be applied to CDS observations. For general definitions and applications on this subject see, e.g., Mason \& Monsignori Fossi (1994) and Del Zanna et al. (2002b). The intensity $I\left(\lambda_{i j}\right)$, of an optically thin spectral line of wavelength $\lambda_{i j}$ can be written:

$I\left(\lambda_{i j}\right)=\int A_{b}(X) C\left(T, \lambda_{i j}, N_{\mathrm{e}}\right) \operatorname{DEM}(T) \mathrm{d} T$

where we have defined the differential emission measure $\operatorname{DEM}(T)=N_{\mathrm{e}} N_{\mathrm{H}} \frac{\mathrm{d} h}{\mathrm{~d} T}, A_{b}(X)$ is the element abundance, and $N_{\mathrm{e}}$ and $N_{\mathrm{H}}\left(\mathrm{cm}^{-3}\right)$ are the electron and hydrogen number densities. The contribution function $C\left(T, \lambda_{i j}, N_{\mathrm{e}}\right)$ of each line contains all the atomic parameters, in particular the ionization fraction. It is peaked in temperature, confining the emission to a limited temperature range.

The conditions in the "quiet" solar corona are such that ionization equilibrium normally holds, and the $C(T)$ s are calculated using ionization balance calculations. These in turn therefore have a significant role in the derivation of the DEM. The CHIANTI package (version 4.01, Young et al. 2003) has been used here to calculate the contribution functions of the observed lines, assuming collisional ionization equilibrium.

The DEM analysis was performed using a modified version of the Arcetri inversion code (Monsignori Fossi \& Landini 1991). Plots of $D E M$ values are not presented in this paper. Instead, we provide examples of the use of the emission measure loci method (see Del Zanna et al. 2002b, and references therein for details). This method consists of plotting the ratios of the observed intensities $I_{\mathrm{ob}}$ and the contribution functions $I_{\mathrm{ob}} /\left(A_{b} * C(T)\right)$ as a function of temperature and use the envelope of these curves to constrain the emission measure distribution and the relative elemental abundances. This method is particularly useful when the plasma emission along the line of sight is nearly isothermal, since in this case all the curves should cross at one point.

The CDS wavelength range is rich in density diagnostics (see Mason et al. 1997), mainly confined to high-temperature lines (above $1 \mathrm{MK}$ ). We provide estimates of the electron densities (averages over the line of sight) using line ratios calculated at the appropriate temperature, and including proton alongside electron collisional processes.

Another parameter which can be determined spectroscopically is the composition of the coronal plasma. Coronal abundances have been found to differ from photospheric values in a way which appears to be related to the first ionization potential (FIP) of the various elements (see the reviews of Fludra \& Schmelz 1999; Raymond et al. 2001). Some results should be treated with caution, given the approximations used and the first-order effects that have often been neglected (see Del Zanna et al. 2001b, 2003).

Finally, the plasma filling factors $f$ can be determined once the total emission measure $E M=\int D E M(T) \mathrm{d} T$ is calculated, the average density $\left\langle N_{\mathrm{e}}>\right.$ is estimated, and the line-of-sight depth $\Delta h$ of the volume of the emitting plasma is measured:

$$
f=\frac{E M}{<N_{\mathrm{e}}>^{2} \Delta h}
$$

Filling factors are difficult to measure because of the many uncertainties in the estimates of emission measures, densities and volumes. The quiescent $1 \mathrm{MK}$ loops turn out to be one of the best cases, because of their simple geometry and temperature structure, as discussed below.

\subsection{Data analysis}

The analysis of TRACE data was performed using the standard routines available within Solarsoft ${ }^{2}$. The main processing is the removal of a dark current and of the energetic particle hits, performed with the program trace prep. A first-order estimate of the uncertainties is calculated assuming Poisson statistics.

The analysis of the CDS data is more complex, and was performed with user-written programs, together with the standard ones available within Solarsoft (details may be found in Del Zanna 1999; Del Zanna et al. 2001a). Line intensities were obtained by using multiple Gaussian line-fitting routines (see Haugan 1997) on the corrected spectra, removing the "continuum" intensity, which is mainly due to scattered light in the NIS.

The CDS calibration of Del Zanna et al. (2001a) has been adopted here, with a correction to take into account the effects on the NIS detector of the gain depression caused by exposures with the wide slit. This calibration is in overall agreement (10-30\%) with the CDS standard calibration (see Del Zanna 2002 and Lang et al. 2002, for details) for most part of the spectra, although some differences can be as high as $50 \%$. For example, regarding the Mg VII density-sensitive 319/367 $\AA$ ratio, the CDS standard calibration gives values about $50 \%$ lower than those presented here.

\section{The CDS and TRACE observations}

We searched the entire TRACE and CDS databases to look for simultaneous CDS and TRACE (173 and $195 \AA$ ) observations of quiescent active regions. Very few cases were found. One of us (HEM) was CDS planner during May 1998 and coordinated a week-long effort to obtain simultaneous CDS and TRACE observations. The CDS observing sequences were designed by Peter Young. On May 2 1998, CDS and TRACE observed an active region on the limb for most of the day. This active region was very quiet during the entire observation period. Here we present results of only the first hour-long set of TRACE and CDS simultaneous observations.

\footnotetext{
${ }^{2}$ http://www.lmsal.com/solarsoft/
} 
Table 1. Principal lines present in the AR_MON CDS-NIS study. Wavelengths are taken from the CHIANTI database. When only one decimal place is indicated, the lines are blends of at least two transitions. Some of the principal blends are listed, as well as the number of transitions within a multiplet. The approximate temperature of maximum ionization fraction is also indicated. The lines used for the EM loci plots in the loop (L) and background (B) regions are marked with a star. Some are not selected because of low intensities or blends. Those in parentheses have been used only in a limited number of cases. The lines used for density diagnostics are marked with a "d" (the Fe XIV 353.8/334.1 $\AA$ ratio is in the low-density limit and has not been used).

\begin{tabular}{|c|c|c|c|c|c|}
\hline Ion & $\begin{array}{l}\lambda \\
(\AA)\end{array}$ & $\begin{array}{l}T_{\max } \\
(\log )\end{array}$ & NIS & $\mathrm{L}$ & B \\
\hline Mg VII (bl Ni XV) & 319.027 & 5.8 & 1 & $\mathrm{~d}$ & \\
\hline Si VIII & 319.825 & 5.9 & 1 & $*$ & $*$ \\
\hline Fe XIII & 320.809 & 6.2 & 1 & & $\mathrm{~d}$ \\
\hline Fe XIII & 321.4 & 6.2 & 1 & & $\mathrm{~d}$ \\
\hline Fe XIV & 334.172 & 6.2 & 1 & & $*$ \\
\hline Fe XII (bl Ca VII ?) & 346.852 & 6.2 & 1 & & \\
\hline Si X & 347.402 & 6.1 & 1 & & $\mathrm{~d}$ \\
\hline Fe XIII & 348.183 & 6.2 & 1 & & $*$ \\
\hline $\operatorname{Mg}$ VI $(4$, bl Fe XI) & 349.2 & 5.7 & 1 & $(*)$ & $(*)$ \\
\hline Fe XIV (bl ?) & 353.831 & 6.2 & 1 & & \\
\hline Si X (2) & 356.0 & 6.1 & 1 & & $\mathrm{~d}$ \\
\hline Fe XI (bl Fe XIV) & 356.5 & 6.1 & 1 & & \\
\hline Fe XIII (2) (bl?) & 359.8 & 6.2 & 1 & & \\
\hline Fe XVI & 360.761 & 6.3 & 1 & & $*$ \\
\hline Mg VII & 363.772 & 5.8 & 1 & & \\
\hline Fe XII & 364.467 & 6.1 & 1 & & $*$ \\
\hline Mg VII (3) & 365.2 & 5.8 & 1 & $*$ & $*$ \\
\hline Mg VII (2) & 367.7 & 5.8 & 1 & $*$ & $*$ \\
\hline Mg IX & 368.070 & 6.0 & 1 & $*$ & $*$ \\
\hline Si XII & 520.665 & 6.3 & 2 & & $*$ \\
\hline $\mathrm{CaX}$ & 557.765 & 5.8 & 2 & & \\
\hline $\mathrm{Ne}$ VI & 558.594 & 5.6 & 2 & $*$ & $*$ \\
\hline $\mathrm{Ne} \mathrm{VII}$ & 561.728 & 5.7 & 2 & $*$ & $*$ \\
\hline $\mathrm{Ne}$ VI (bl) & 562.803 & 5.6 & 2 & $*$ & $*$ \\
\hline Si XI (bl ?) & 563.957 & 6.2 & 2 & & \\
\hline $\mathrm{He} \mathrm{I}$ & 584.350 & 4.5 & 2 & & \\
\hline O III & 599.597 & 5.1 & 2 & & \\
\hline $\operatorname{Mg} X$ & 624.941 & 6.0 & 2 & $*$ & $*$ \\
\hline O V (bl Mg VIII IIo) & 629.732 & 5.4 & 2 & $(*)$ & $(*)$ \\
\hline
\end{tabular}

TRACE observed the AR with both 173 and $195 \AA$ bands, alternating between the two, with a cadence of 2 min (i.e every 2 min two exposures in the two bands were recorded). The exposure times were 27.56 and $23.17 \mathrm{~s}$ for most exposures, in the 173 and $195 \AA$ bands respectively, leading to data numbers (DN) per pixel of the order of 100 in the active region.

The CDS observation consisted of a NIS raster (CDS study AR_MON) formed by scanning with the $4^{\prime \prime} \times 240^{\prime \prime}$ slit, over a period of about an hour. The plate scale at the CCD is such
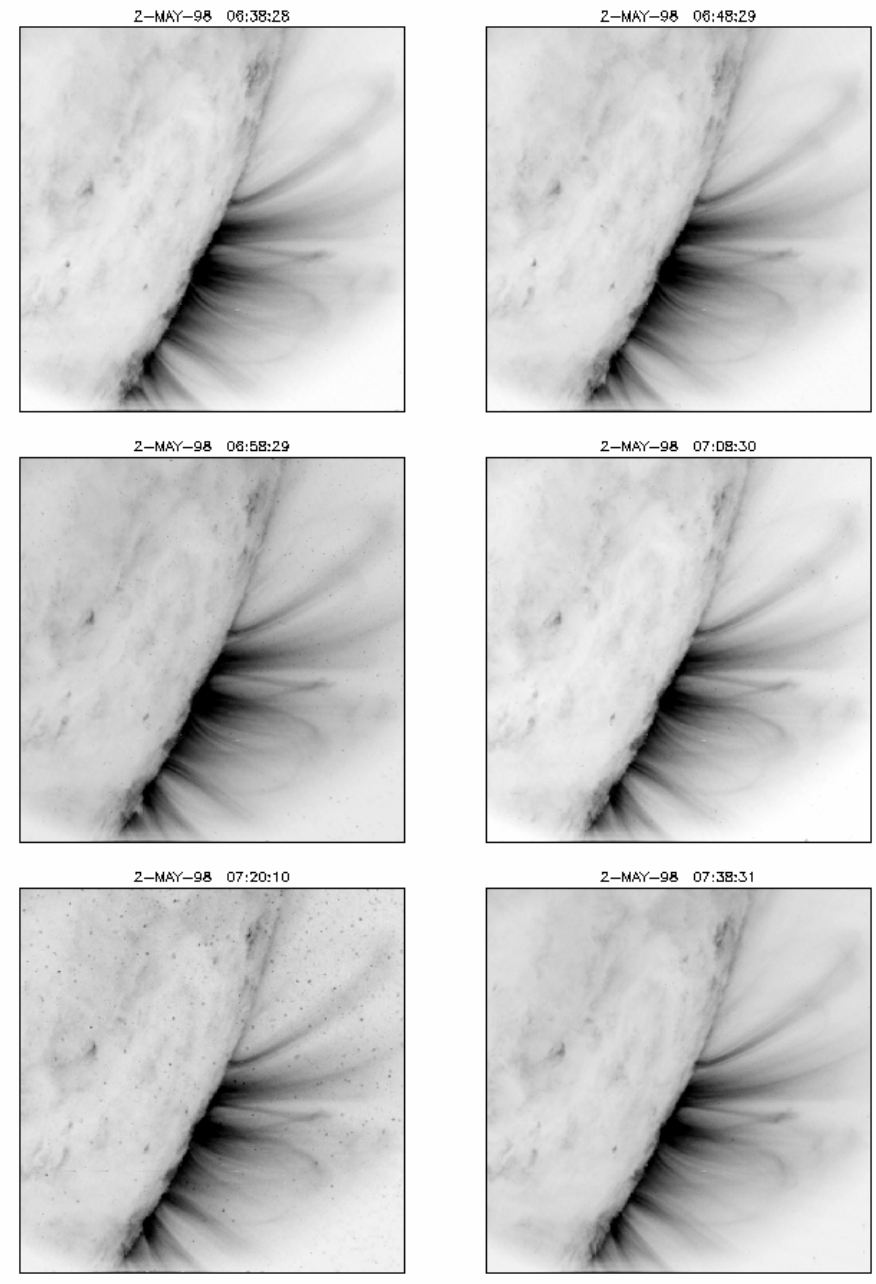

Fig. 1. Sequence of TRACE $173 \AA$ negative images of the active region. Many loops are visible. The images are plotted with the same intensity scale, and show that the loops were stationary during the whole period of the CDS observation.

that a pixel is equivalent to $1.68^{\prime \prime}$ along the slit. The study was designed to rebin the spectra by a factor of two along the slit, giving a scale of $3.36^{\prime \prime}$, still lower than the instrument resolution $\left(5-7^{\prime \prime}\right)$.

The NIS observation started at 6:38 UT and ended at 7:40 UT. The area of the raster was approximately $4^{\prime} \times 4^{\prime}$, with an exposure time of $47 \mathrm{~s}$ for each exposure. The NIS extraction windows contained a large selection of lines covering a wide range of temperatures (see Table 1).

Figure 1 displays a sample sequence of TRACE 173 images, showing the presence of a system of quiescent $1 \mathrm{MK}$ loops. These loops were in fact very stable during the whole period of the CDS raster. Difference images only show small variations, at maximum of the order of $10 \%$. Larger differences were observed at the limb, but not off-limb. The active region was also very stable as seen in the $195 \AA$ band.

Monochromatic images were obtained from the NIS data and are are shown in Fig. 2. This active region presents the typical characteristics of the quiescent active regions observed by CDS. The maximum visibility of the loops is in the upper transition region lines such as $\mathrm{Mg}$ VII, Ca X, Mg IX. These 

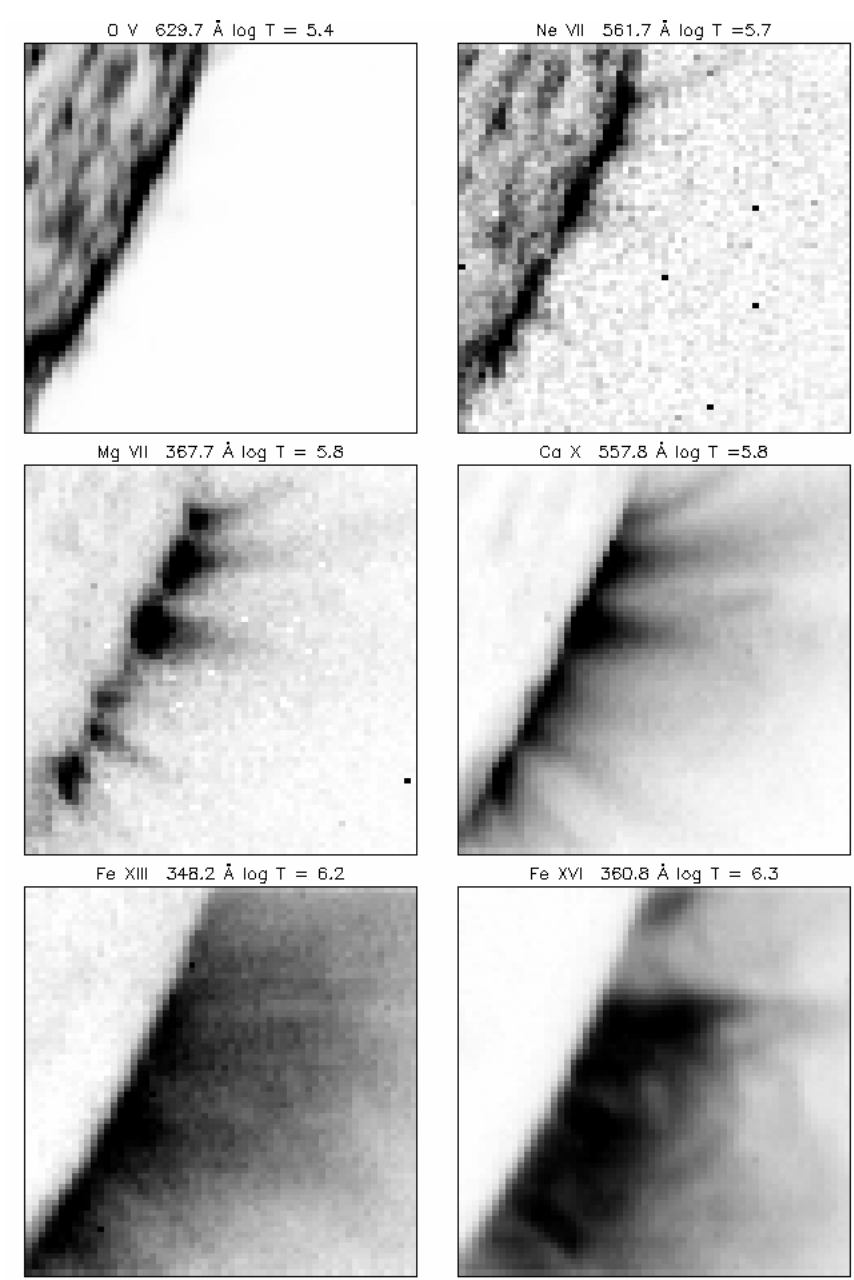

Fig. 2. Monochromatic images (negative) of the NIS observation. The loops are only seen in upper transition region lines (Mg VII, Ca X) emitted in the range $\log T=5.8-6.0$, a clear indication of their isothermality.

loops are not visible in high-temperature (e.g. Fe XII) lines, nor in cooler TR lines. At higher temperatures, between 1.5 and $2 \mathrm{MK}$, the active region produces only diffuse background emission. At even higher temperatures low-lying hot loops are present, as seen in e.g. Fe XVI. These loops do not have any direct spatial relationship with the $1 \mathrm{MK}$ loops, but are the loops that are normally observed with e.g. Yohkoh/SXT. Yohkoh/SXT did not observe this active region simultaneously, but full-Sun images before and after the CDS observation are available. The images in the AlMg filter show exactly the same structures seen in Fe XVI, and also show that this active region was quiescent at these hotter temperatures for at least a few hours.

For a direct comparison with the CDS raster, two averaged TRACE images (one for each bandpass) have been constructed, by taking five exposures in the time range 7:04-7:12 UT, i.e. centred around the time when the CDS slit was positioned near the leg of the northernmost loop, which is discussed in detail in Sect. 3.1 below. The TRACE and CDS observations have been accurately co-aligned, taking into account the differences between the spatial scales as seen from the SOHO and
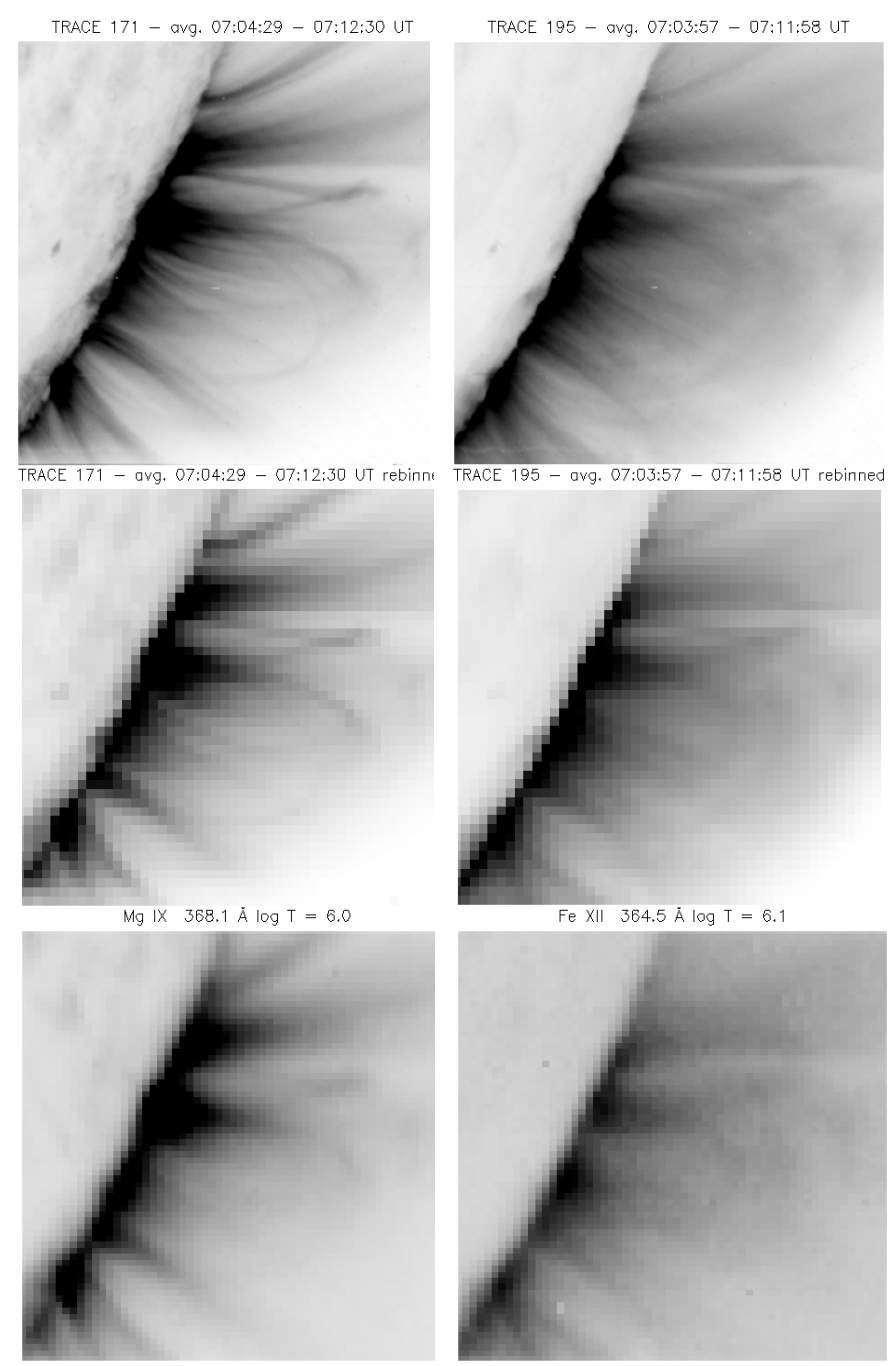

Fig. 3. From top to bottom: TRACE 173 and $195 \AA$ A negative images of the active region; the same images rebinned by a factor of 10 (each pixel size $=5^{\prime \prime}$ ); the CDS images in Mg IX and Fe XII; Note: 1) the strong similarities between the TRACE $173 \AA$ and CDS Mg IX, which confirm: a) the lines that contribute to the $173 \AA$ band are emitted over similar temperatures as Mg IX; b) the CDS spatial resolution is approximately $5^{\prime \prime} .2$ ) The almost complete absence of loop emission in the TRACE $195 \AA$ image. 3) The presence of structures in the TRACE $195 \AA$ A image that cannot be attributed to Fe XII emission.

TRACE viewpoints. The TRACE images have been rescaled to $\mathrm{L} 1$, where $\mathrm{SOHO}$ is located. The CDS pointing was very accurate, as confirmed by the location of the solar limb as seen in He I. The TRACE pointing was also good, and only required an adjustment of $3^{\prime \prime}$. This was obtained by alignment of distinct features (solar limb, loops) as seen in the $173 \AA$ band and in the CDS coronal lines.

Figure 3 shows the TRACE 173 and $195 \AA$ images of the CDS field of view, together with the CDS images in Mg IX and Fe XII. The latter images should show exactly the same features of the two TRACE bands, if these are dominated by Fe IX-X and Fe XII emission. However, some differences are to be expected because of the different spatial resolutions (a CDS pixel is $4.04^{\prime \prime} \times 3.36^{\prime \prime}$ while a TRACE pixel is $0.505 \times 0.505^{\prime \prime}$ 
at L1). For a more direct comparison, Fig. 3 also shows the TRACE images rebinned to a 5.05" resolution.

We derive quantitative results in later sections, but first we stress some important points that can be deduced immediately from the simple direct comparison between the CDS and the TRACE images:

1) the 1 MK loops seen in TRACE $173 \AA$ must be almost isothermal along the line of sight, otherwise they would be visible in CDS lines emitted over a broad range of temperatures. There is excellent consistency between the TRACE $173 \AA$ image and CDS images in lines emitted at 0.8-1.0 MK (e.g. Mg IX);

2) these $1 \mathrm{MK}$ loops are much cooler than the surrounding diffuse emission, since they are visible in CDS lines emitted at lower temperatures;

3 ) these quiescent $1 \mathrm{MK}$ loops, as normally the case in TRACE images, are clearly visible in the $173 \AA$ filter, but not in the $195 \AA$ one, where the emission is more diffuse. This immediately raises a question about the applicability of the filter-ratio method. In fact, for this method to produce meaningful values, not only must the plasma be isothermal along the line of sight, but also the emission recorded in the two bands must have been produced by a plasma that is co-spatial;

4) the diffuse background along the line of sight represents a significant fraction of the loop emission (examples are given below, see e.g. Fig. 5);

5) some of the $1 \mathrm{MK}$ loops are partially visible in the TRACE $195 \AA$ filter. However, the signal recorded by TRACE in this band cannot always be due to Fe XII emission, otherwise all the loops would be clearly visible by CDS in Fe XII lines or in ions emitted at similar temperatures. Below we argue that in some cases the $195 \AA$ signal is composed of a superposition of diffuse AR background and of Fe VIII emission originating only from the $1 \mathrm{MK}$ loops;

6) the CDS spatial resolution is approximately 5-7", and not larger as some authors have claimed (e.g. 10-15" in Aschwanden 2002);

7) with the TRACE resolution, most loop structures appear to be composed of multiple threads. The loops visible in CDS are therefore a superposition of several threads, as seen in TRACE; 8) there are almost no regions free of AR loop emission, which can be used for a proper background subtraction. This holds for both TRACE and CDS, and represents a severe limitation, in particular for off-limb datasets. One exception is discussed below.

\subsection{A closer look at the loop leg}

We have selected the long loop that extends northward of the active region for detailed analysis. This loop, clearly visible in the TRACE $173 \AA$ image (see also Fig. 13), is a rather long one, with a projected semi-length of approximately $150 \mathrm{Mm}$. CDS only observed the leg of this loop, up to about $1 / 3$ of its length. Moreover, it is difficult to assess the exact location of its footpoint, probably very close to the solar limb. However, spectroscopic observations of loop legs are important, since the TRACE passbands cannot provide any useful information

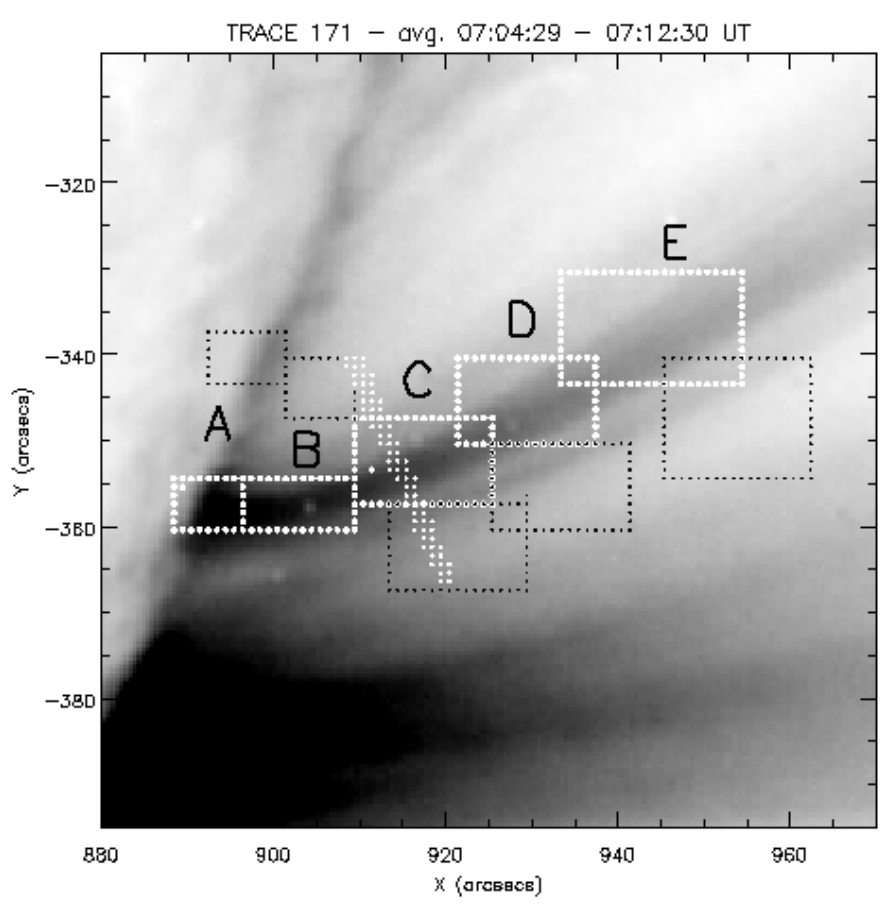

Fig. 4. The areas selected to obtain average spectra in the loop (marked in white) and in the background (marked in black), projected on the TRACE image. Also shown is the area selected to show profiles of count rates across the loop.

at transition region temperatures. There is a sufficient spatial overlap between the CDS and TRACE observations to allow a direct comparison over the loop leg and its adjacent diffuse background region. The main uncertainty lies in the selection of suitable background regions close to the limb, for both instruments.

The TRACE $173 \AA$ images show that the loop is composed of at least three strands, a main one in the centre and one on either side which only contribute a fraction of the total emission (see Fig. 5). The width of the main strand slightly increases with height. To estimate the width of the strand we can reasonably assume a cylindrical shape. If we assume a constant density inside the strand and optically thin emission, we can calculate the predicted intensity profile across the loop, as a function of the loop radius and with an adjustable constant factor. We find a good agreement between the $173 \AA$ profiles and widths ranging between $5^{\prime \prime}$ and $15^{\prime \prime}$ (see Fig. 5 for an example). CDS did not resolve the three strands observed by TRACE, but clearly resolved the overall loop structure.

In order to reduce the effects of energetic particle hits and increase the signal-to-noise, five spatial positions have been selected for subsequent averaging (for both CDS and TRACE) along the loop leg region (A, B, C, D, E shown in Fig. 4). These regions contain only a few $\mathrm{CDS}$ pixels each, but a large number of co-spatial TRACE pixels. Obviously, the TRACE areas are not optimal, since they also include some background (a better selection is provided later in Sect. 4.1). The background regions were selected near the loop regions, in areas free of contamination. This was simple for regions $\mathrm{C}, \mathrm{D}, \mathrm{E}$, while it is obviously difficult to define appropriate background areas A and B. Results related to the latter background areas should therefore 
Table 2. CDS and TRACE measurements concerning the selected regions (A-E), in the loop leg (Loop), the adjacent background areas (Backgr.), and the corresponding background-subtracted values. The averaged distances along the loop (starting from the limb, where region A is) are shown in the first row. Electron densities $N_{\mathrm{e}}\left(10^{8} \mathrm{~cm}^{-3}\right)$ have been derived from the averaged CDS spectra and the Del Zanna et al. (2001a) calibration. Temperatures, derived from the $D E M$ method, and total emission measures $E M=\int D E M(T) \mathrm{d} T$ are also shown. The bottom part shows the averaged TRACE count rates $\left(\mathrm{DN} \mathrm{s}^{-1} \mathrm{pix}^{-1}\right)$ in the two bands, $I(173 \AA$ ) and $I(195 \AA)$, together with their ratios.

\begin{tabular}{|c|c|c|c|c|c|c|}
\hline & A & B & $\mathrm{C}$ & $\mathrm{D}$ & $\mathrm{E}$ & Region \\
\hline Distances along the loop $\left(^{\prime \prime}\right)$ & 0 & 8 & 25 & 40 & 60 & \\
\hline$N_{\mathrm{e}}(\mathrm{Mg}$ VII $)$ & $32 \pm 12$ & $26 \pm 6$ & $11 \pm 8$ & $13 \pm 6$ & $8 \pm 8$ & Loop \\
\hline$N_{\mathrm{e}}(\mathrm{Mg}$ VII $)$ & $38 \pm 19$ & $24 \pm 15$ & $4 \pm 4$ & $3 \pm 3$ & $2.5 \pm 2.5$ & Loop (backgr. sub.) \\
\hline$N_{\mathrm{e}}(\mathrm{Si} \mathrm{X} \mathrm{356/347.4} \mathrm{\AA )}$ & $9 \pm 1$ & $6.7 \pm 0.8$ & $6.3 \pm 0.5$ & $5.6 \pm 0.6$ & $5.3 \pm 0.6$ & Backgr. \\
\hline$N_{\mathrm{e}}(\mathrm{Fe}$ XIII $320.8 / 321.4 \AA)$ & $9 \pm 1$ & $7.9 \pm 0.2$ & $8.9 \pm 2$ & $6.7 \pm 1$ & $6.3 \pm 1$ & Backgr. \\
\hline$N_{\mathrm{e}}(\mathrm{Fe}$ XIII $320.8 / 348.183 \AA)$ & $11 \pm 2$ & $8.9 \pm 1$ & $7.5 \pm 1$ & $7.5 \pm 0.9$ & $6.0 \pm 0.7$ & Backgr. \\
\hline $\log T_{\mathrm{e}}(\mathrm{K})$ & 5.83 & 5.87 & 5.9 & 5.92 & 5.95 & Loop (backgr. sub.) \\
\hline$E M\left(10^{26} \mathrm{~cm}^{-5}\right)$ & 19 & 11 & 4.9 & 1.4 & 0.5 & Loop (backgr. sub.) \\
\hline$I(173 \AA)$ & $7.0 \pm 0.5$ & $7.0 \pm 0.5$ & $5.6 \pm 0.4$ & $4.5 \pm 0.4$ & $3.5 \pm 0.3$ & Loop \\
\hline$I(195 \AA)$ & $6.7 \pm 0.6$ & $6.6 \pm 0.5$ & $5.2 \pm 0.5$ & $4.5 \pm 0.4$ & $3.8 \pm 0.4$ & Loop \\
\hline$I(173 \AA)$ & $3.4 \pm 0.3$ & $3.2 \pm 0.3$ & $4.0 \pm 0.4$ & $3.0 \pm 0.3$ & $2.1 \pm 0.3$ & Backgr. \\
\hline$I(195 \AA)$ & $3.3 \pm 0.4$ & $4.9 \pm 0.5$ & $5.2 \pm 0.5$ & $4.3 \pm 0.4$ & $3.5 \pm 0.4$ & Backgr. \\
\hline$I(195 \AA) / I(173 \AA)$ & $0.94 \pm 0.23$ & $0.45 \pm 0.20$ & - & $0.13 \pm 0.13$ & $0.21 \pm 0.21$ & Loop (backgr. sub.) \\
\hline$I(195 \AA) / I(173 \AA)$ & $0.96 \pm 0.10$ & $0.94 \pm 0.10$ & $0.93 \pm 0.11$ & $1.0 \pm 0.13$ & $1.09 \pm 0.17$ & Loop \\
\hline
\end{tabular}

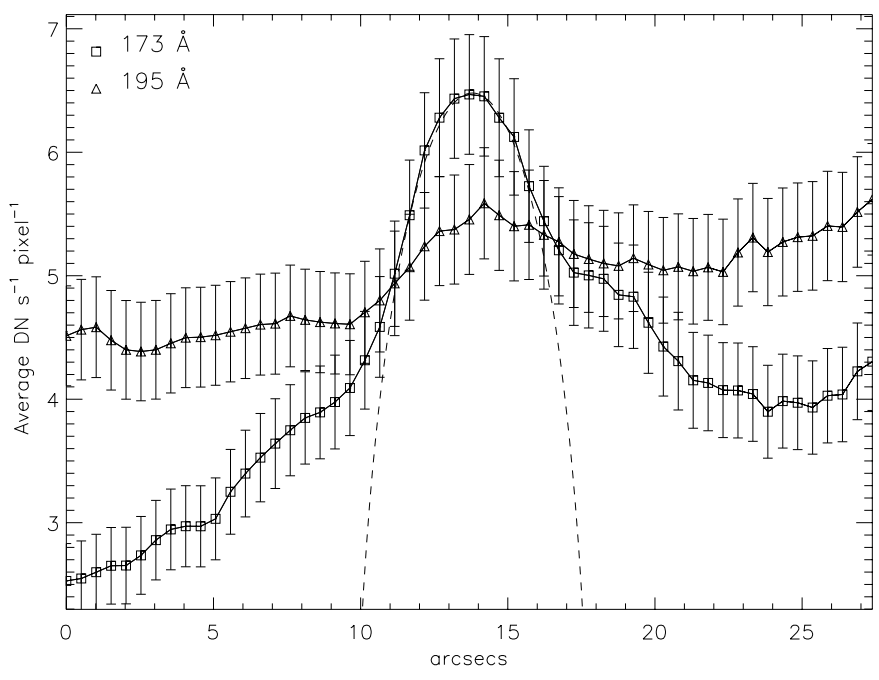

Fig. 5. The profile of the TRACE count rates across the loop, close to area C (see Fig. 4). Over-plotted with a dashed line is the $173 \AA$ intensity profile that would be expected from a circular cross-section with constant density and a width of $8^{\prime \prime}$. Note the small difference in the loop and background signal, in particular for the $195 \AA$ band.

be regarded as the most uncertain. The CDS and TRACE results associated with the five regions are shown in Table 2.

CDS line intensities have been obtained from the averaged spectra, with multiple line fitting and removal of the "continuum" emission. A sample of a few NIS averaged spectra for region B are displayed in Fig. 6. The signal in each averaged spectrum is very good. It is obvious that the loop has increased emission only in lines emitted at temperatures 0.7-1 MK (such as Mg VII, Si VIII, Mg IX). In hotter lines (such as Fe XII), the coronal emission is mainly due to the AR diffuse background.
Also note that even in the lines where the loop is visible, the contribution from the diffuse background is significant.

\subsubsection{Densities and temperatures of the loop and background}

First, we want to measure the electron density, inside and outside the loop. In the diffuse background regions, this is relatively straightforward, since a number of diagnostic line ratios are available (Si X, Fe XIII). The results are displayed in Table 2. Obviously, these values are averaged ones along the line of sight, which is quite large (the extent of the active region), but should be fairly close to the actual values near the loop. In fact, there are no obvious inhomogeneities in the background emission and the density is expected to fall off exponentially in the radial direction (a refined estimate would require inversion of the Abel integral and some assumptions). Note the good agreement between the densities derived from different line ratios and ions and the fact that the densities are about a factor of 2 higher than the corresponding values in the quiet Sun (see Del Zanna 1999; Fludra et al. 1999).

For the loops, we have the problem that the intensities of most of the density-sensitive lines are, within uncertainties, the same in the loop and background regions. The only exception are the Mg VII lines. The Mg VII $319 \AA$ line can be used to estimate densities, in conjunction with one of the other $\mathrm{Mg}$ VII lines. It is normally a rather weak line, but since most of the emission of the loop leg comes from plasma at temperatures where Mg VII is most abundant, this line is easily detectable in the spectra close to the base of the loop (see Fig. 6, top left). To isolate the emission originating only from the loop we need to subtract a background intensity, which significantly 

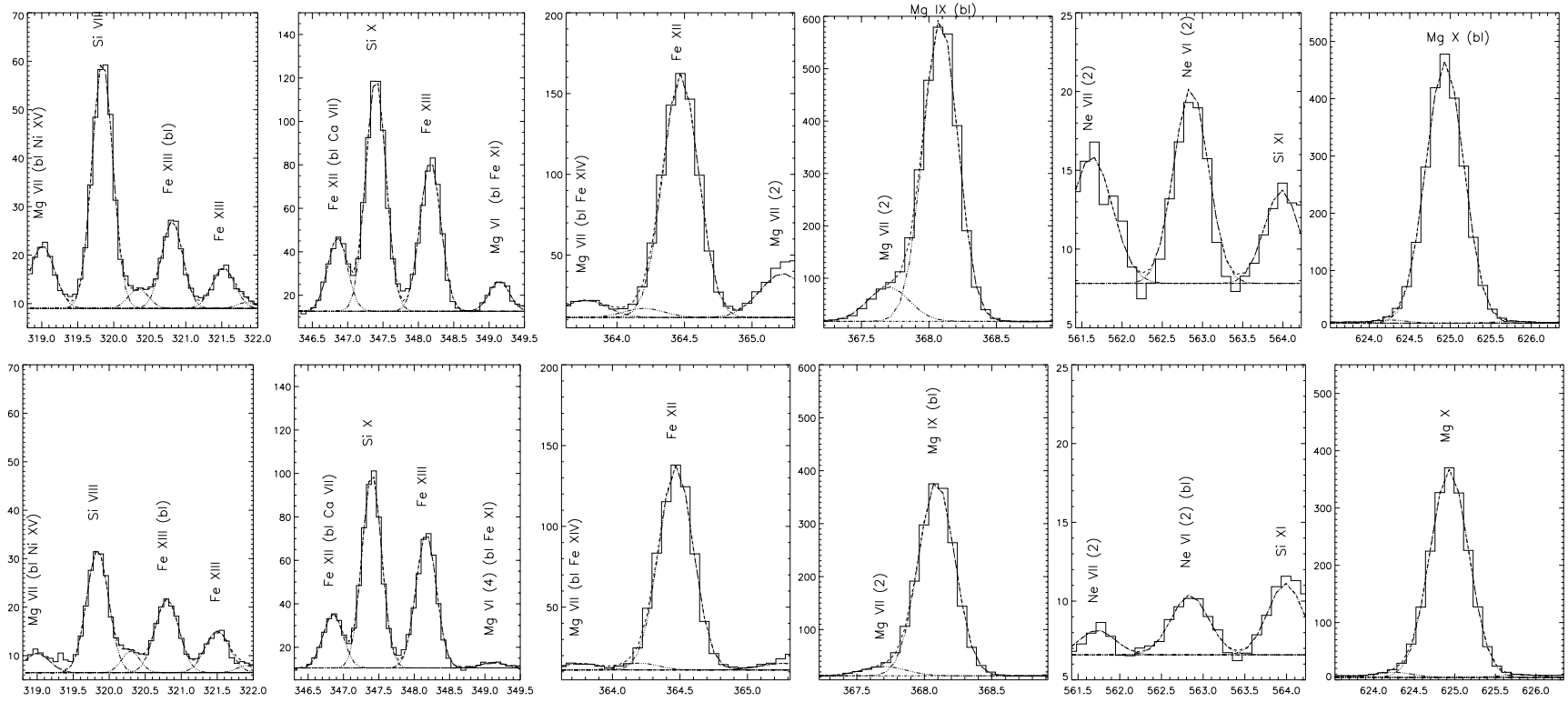

Fig. 6. Sample averaged spectra (photon-events vs. wavelengths in $\AA$ ) for the loop area (above) and background (below) of region B (see Fig. 4). Note that the intensity of the spectral lines formed above $1 \mathrm{MK}$ are almost the same in the loop and background regions, indicating that most of the emission is in the background. Also note in the loop area the large increase in the emission of lines formed in the 0.7-0.9 MK range (e.g.: Mg VII, Si VIII, Mg IX). The lines shown have been used for density, emission measure and elemental abundance estimates.

increases the uncertainties. We provide in Table 2 both density estimates, calculated by subtracting (or not) the background intensities. Accurate measurements are difficult, mostly because of the uncertainty in removal of both the pseudo-continuum in the NIS spectra, and of the background intensities. However, the indication is for high densities $\left(N_{\mathrm{e}} \simeq 3 \times 10^{9} \mathrm{~cm}^{-3}\right)$ near the base, and a rapid decrease with height.

Now a word of caution about the use of Mg VII 319.0 $\AA$. This line can be blended with a Ni XV, if there is very "hot" emission along the line of sight. We have performed DEM analyses on the line intensities of various regions of the CDS raster and found that in the places where the Fe XVI emission is very high (in most of the AR but not where the loop analysed here is located) the Ni XV line can contribute about $25 \%$ to the blend.

Second, we want to estimate the temperature distribution of the plasma along the line of sight, in both the loop leg and the adjacent background regions. We have performed both the DEM and the emission measure loci methods, using a selection of lines that are approximately density-insensitive (see Table 1).

The $I_{\mathrm{ob}} /\left(A_{b} * C(T)\right)$ curves, calculated with the coronal abundances of Feldman (1992), and a density $N_{\mathrm{e}}=7 \times$ $10^{8} \mathrm{~cm}^{-3}$ and the ion fractions of Arnaud \& Rothenflug (1985) are displayed in Fig. 7. The emission measure loci method clearly show that:

1) the plasma in the loop leg is isothermal, since all the curves cross at one point;

2) the emission measures peak at upper transition region temperatures $(\mathrm{Mg} \mathrm{VII}, \mathrm{Ca} \mathrm{X})$, with a rapid decrease from 0.9 to $0.7 \mathrm{MK}$ toward the footpoint;

3) the thermal distribution in the diffuse background emission is very different. Mainly because is not isothermal along the line of sight, with a flat emission measure distribution at higher temperatures (1-2 MK), typical for an active region.

We have performed various tests and came to the conclusion that these results are quite robust, since they do not appreciably change if different ion fractions (e.g. Mazzotta et al. 1998 ) or densities are used. The DEM analysis produces the same results.

It is interesting to note that the thermal characteristics of the loop leg are quite similar to those of the coronal hole plume bases (see Del Zanna \& Bromage 1999; Del Zanna et al. 2003). In fact coronal hole plumes are also isothermal, cooler than their background, and have emission measures that peak at the same temperatures.

\subsubsection{Elemental abundances and filling factors}

Next, we estimate the relative elemental abundances between low- and high-FIP elements. Here we have the additional problem that at the loop temperatures only a few high-FIP ions emit at the CDS wavelengths. Basically, only Ne VII and Ne VI. These ions produce lines in the NIS spectra that are only observable close to the base of the loop (see Fig. 6), where the density is higher. In these areas, a FIP effect of 3-4 has been measured, using both DEM and emission measure loci methods. This FIP effect is consistent with the coronal abundances of e.g. Feldman (1992) and Fludra \& Schmelz (1999) where the relative low-FIP vs. high-FIP abundances are increased by a factor of about 4, compared to their photospheric ratio values (the main difference lying in the absolute scaling). The Ne VI and Ne VII EM loci curves are also displayed in Fig. 7 and show good agreement with those of Mg VI, Mg VII.

It is also important to know the elemental composition of the coronal plasma in absolute terms (i.e. relative to hydrogen). 


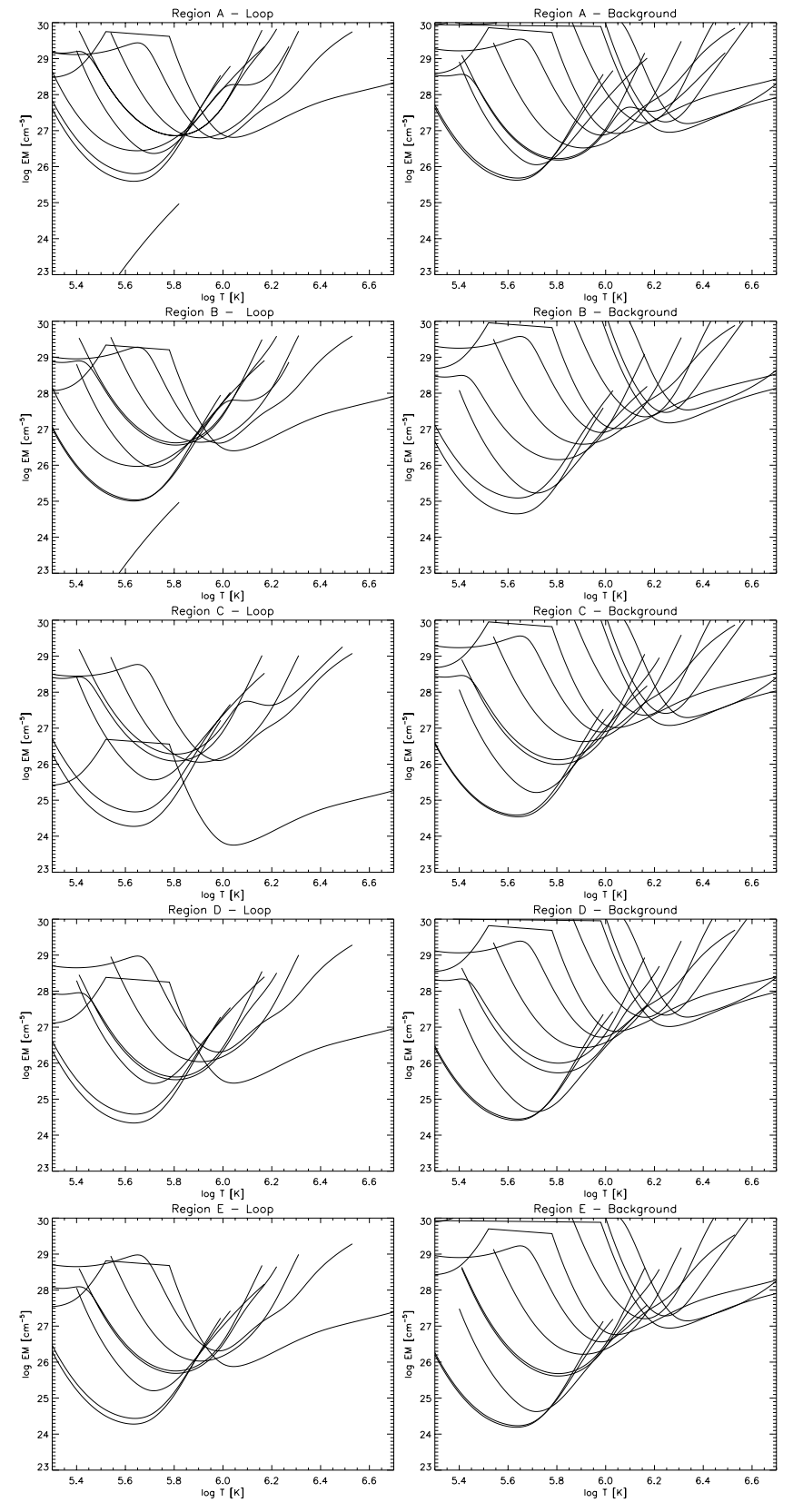

Fig. 7. The emission measure curves obtained from the backgroundsubtracted line intensities of the loop (left) and those of the background (right), for the regions A-E. The ionization equilibrium calculations of Arnaud \& Rothenflug (1985) have been used, with the coronal abundances of Feldman (1992). The lines plotted here are listed in Table 1. The curves in loop regions A, B with lowest $T$ are due to $\mathrm{O}$ V. They should be regarded as upper limits, since this line in off-limb spectra is blended (see Del Zanna et al. 2001a).

CDS does not allow such a direct measurement. However, it is interesting to note that accurate measurements of densities, emission measures and volumes of isothermal plasmas in principle allow an indirect measurement for a lower limit on the absolute abundances of the low-FIP ions (since the filling factor cannot be greater than one). This is not true for multi-thermal plasmas, where it is difficult to uniquely define an emission measure corresponding to specific temperatures and densities.
The $D E M$ analysis of region B produces a total $E M=1.1 \times$ $10^{27} \mathrm{~cm}^{-5}$, using the coronal abundances of Feldman (1992). The estimates of the depth of the loop and the averaged density are $8^{\prime \prime}\left(5.8 \times 10^{8} \mathrm{~cm}\right)$ and $2.2 \times 10^{9} \mathrm{~cm}^{-3}$, giving $<N_{\mathrm{e}}>^{2}$ $\Delta h=3 \times 10^{27} \mathrm{~cm}^{-5}$, i.e. a filling factor close to unity (0.3). Region A has a similar value, while the others have unphysical filling factors (values greater than one), an indication of the fact that the densities obtained from the subtracted line intensities are probably underestimated. For regions $\mathrm{A}$ and $\mathrm{B}$, the absolute abundances of the low-FIP ions could be at most reduced by a factor of 3 (i.e. to values close to the photospheric ones), which would produce a maximum filling factor of 1 .

\section{The TRACE filter ratio method}

Having established the main characteristics of the 1 MK loops, we now discuss the applicability of the filter ratio method to TRACE observations of these types of loops. Any filter ratio method relies on complete and accurate atomic data, both in terms of atomic processes within each ion, and in terms of the ionization balance. The largest uncertainties are currently related to ion balance calculations. In particular, many ions including those from the $\mathrm{Li}$ and $\mathrm{Na}$ isoelectronic sequences have demonstrated an anomalous behaviour, with large departures between predicted and observed emissivities. This problem has been known for more than 30 years but has been largely ignored (cf. Del Zanna et al. 2002b).

For an assessment of the filter ratio method it is important to know which spectral lines are the main contributors to the TRACE loop observations. We do not have direct spectral measurements of the TRACE bands in this case, but we have found that the AR 1 MK loops have strong similarities with the coronal hole plumes. It is therefore reasonable to expect a similar situation to the one described by Del Zanna et al. (2003), where near-simultaneous CDS-GIS and EIT observations of a plume were analysed. They found that Fe VIII emission was the dominant contributor to the EIT $195 \AA$ passband. In the quiet Sun or in the diffuse background emission the situation is quite different, since the main contribution to the EIT $195 \AA$ filter is from Fe XII lines, mixed with some Fe X, Fe XI and Fe XIII emission.

To show that Fe VIII emission is also very important for the TRACE $195 \AA$ observations of the 1 MK loops discussed here, we have taken the $D E M$ of region C (background-subtracted), and calculated a theoretical spectrum using the same parameters (CHIANTI v.4, $N_{\mathrm{e}}=7 \times 10^{8} \mathrm{~cm}^{-3}$, coronal abundances of Feldman 1992, and the ionization fractions of Arnaud \& Rothenflug 1985). Note that the $D E M$ of region C is highly peaked at $\log T(\mathrm{~K})=5.9$. The results for the 173 and $195 \AA$ bands are displayed in Figs. 8 and 9 (top spectra). Then, we have folded these spectra through the TRACE effective areas (ground calibration), obtaining the results shown in the bottom spectra.

A few points are worth noting. First, that the dominant contribution to the $173 \AA$ band comes from Fe IX and Fe X lines, as expected. Second, that for the $195 \AA$ band most of the emission is apparently due to Fe VIII, and not to Fe XII. This result is very similar to that one obtained for EIT and the coronal 

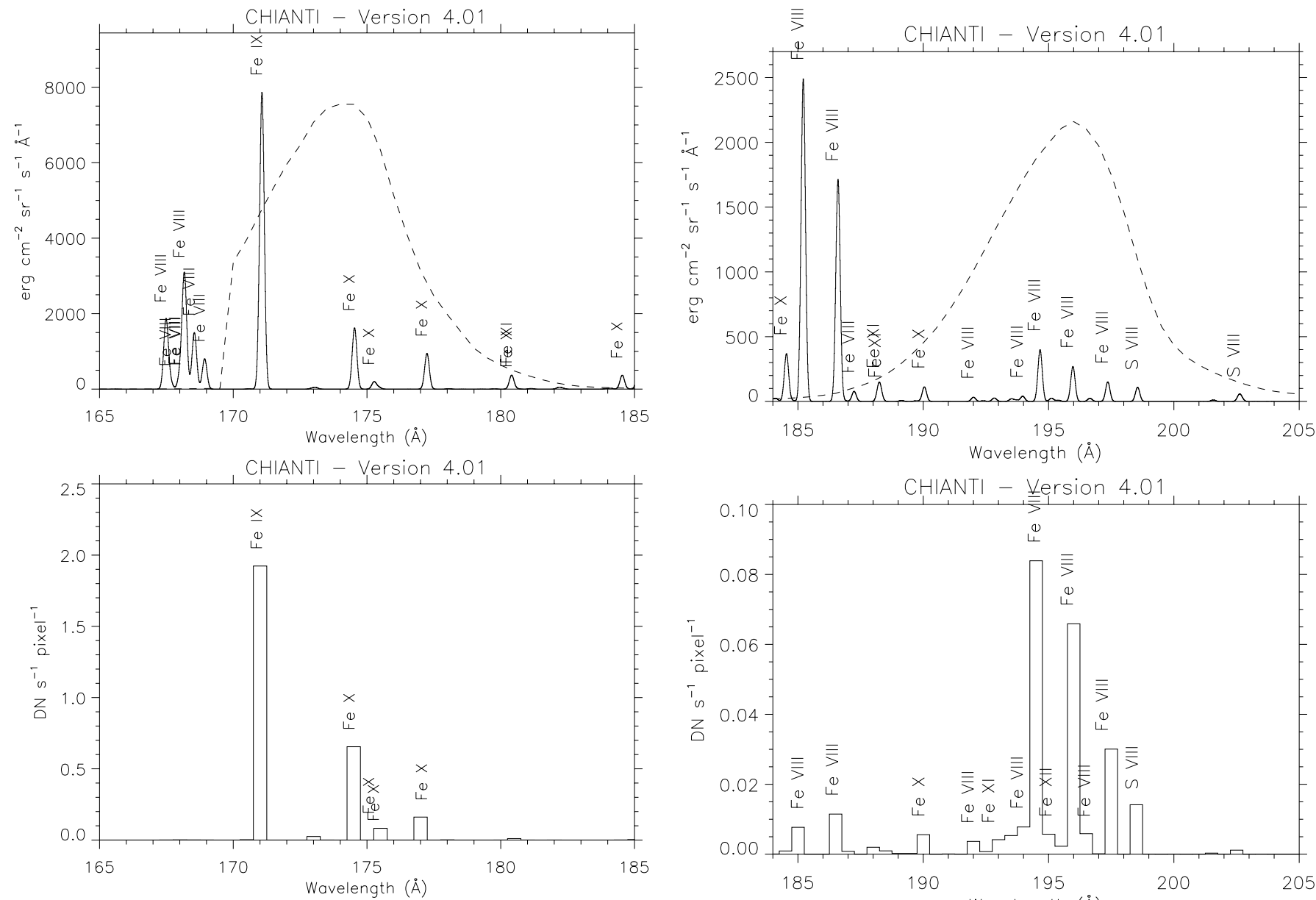

Fig. 8. Top: simulated spectra for the region $\mathrm{C}$ (backgroundsubtracted) in the TRACE $173 \AA$ band (the TRACE effective area, rescaled, is shown with a dashed line). Bottom: simulated spectra folded with the TRACE effective area.

hole plumes by Del Zanna et al. (2003). Third, that the total predicted count rate for the $173 \AA$ band is 2.9 , in overall agreement with the background-subtracted measured value of 1.6 (see Table 2). Bearing in mind that such extrapolations have large uncertainties (say 50\%), this results gives us confidence that the TRACE absolute ground calibration is roughly consistent with the CDS in-flight one.

On the other hand, the situation for the background regions is completely different. There, the DEMs show a flat distribution at coronal temperatures, the total EMs are an order of magnitude higher (mostly because of the much longer path lengths), and the simulated spectra show that the dominant contributors to the TRACE 173 and $195 \AA$ bands are Fe IX/Fe X and Fe XII lines, respectively.

The fact that Fe VIII is important for the TRACE $195 \AA$ bandpass in observations of $1 \mathrm{MK}$ loops has two important consequences for our discussion.

1) the Fe VIII emissivity should be included when calculating the TRACE response function;

2) there is an added uncertainty in the predicted TRACE response function for the TRACE $195 \AA$ bandpass, because of the uncertainty in the ionization fractions of Fe VIII.

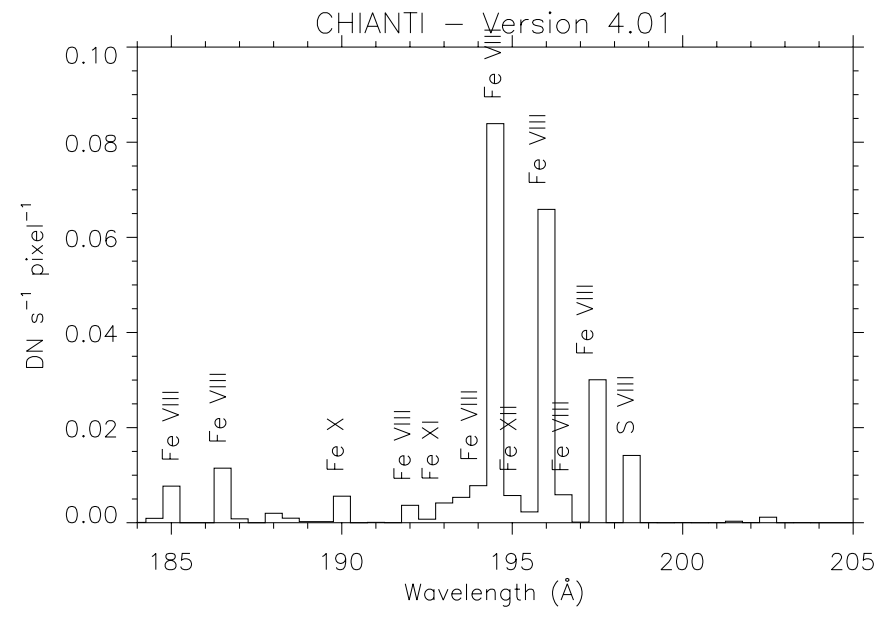

Fig. 9. Same as Fig. 8 for the $195 \AA$ band.

Regarding 1), we note that all the results based on the TRACE temperature sensitivities (which includes the filter ratio method) should be revised since the TRACE response functions available to the community via Solarsoft were calculated (Warren 2002, priv. comm.) with the old version 2.0 of the CHIANTI database (Landi et al. 1999), which did not include the Fe VIII lines emitted in the $195 \AA$ bandpass (because at that time no atomic data was available).

Regarding 2), we note that Fe VIII is one of the anomalous ions (see Del Zanna 1999; Del Zanna et al. 2003). This could in part be due to unreliable calculations of the recombinations from Fe IX. Figure 10 shows the large differences between the Fe VIII and Fe IX ionization fractions calculated by various authors.

We have calculated the new TRACE responses (Fig. 11) using the latest CHIANTI v. 4 (Young et al. 2003), and a set of standard parameters that are the default values in the TRACE analysis software available via Solarsoft (AO filters; ground calibration as described in Handy et al. 1999; ionization balance of Mazzotta et al. 1998; coronal abundances of Feldman 1992; a constant pressure of $1 \times 10^{15} \mathrm{~cm}^{-3} \mathrm{~K}$ ). The new responses and a set of programs to calculate them directly via the CHIANTI software will be provided to Solarsoft. We have then calculated the TRACE 195/173 $\AA$ ratios using different 

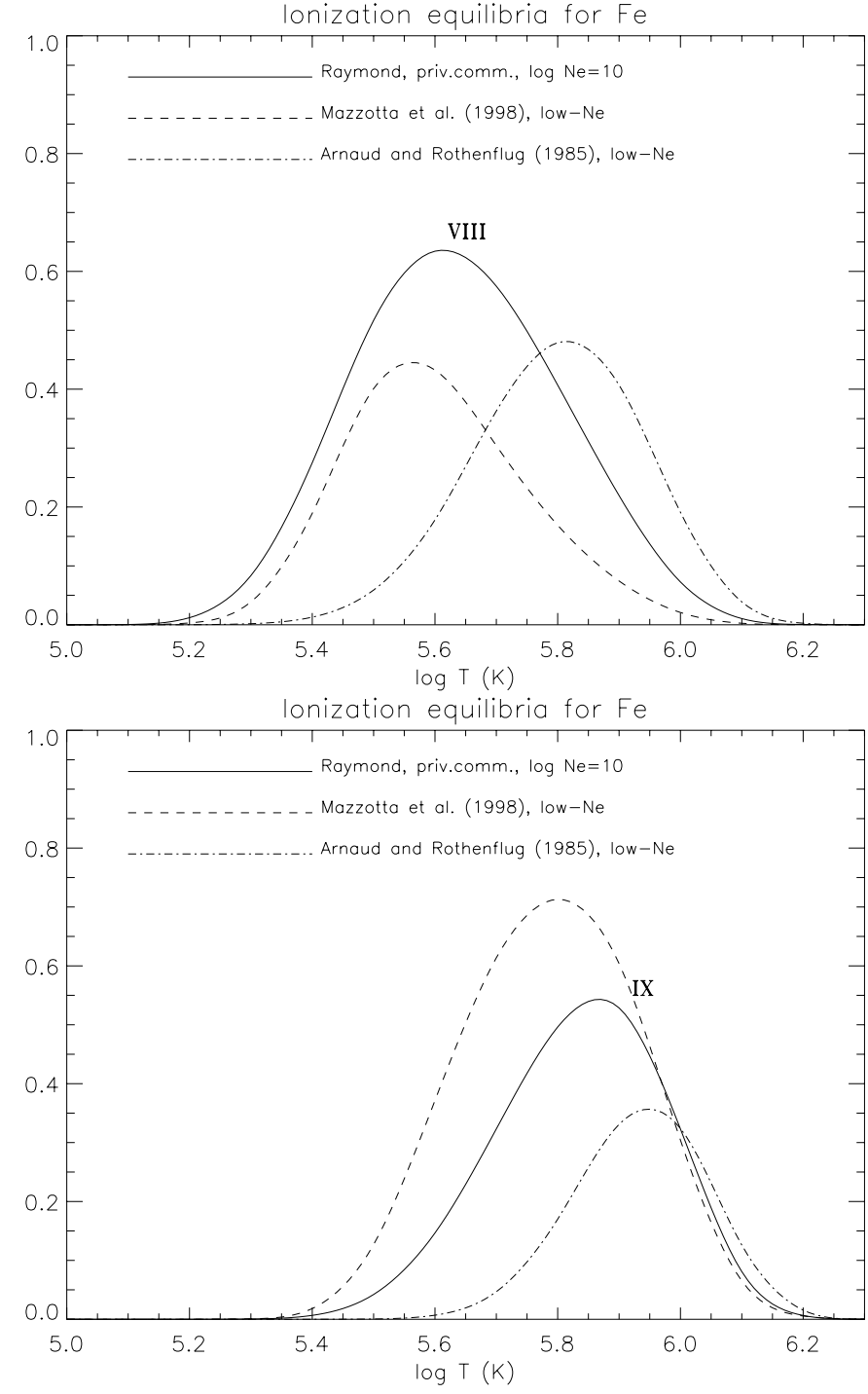

Fig. 10. A comparison of various ionization equilibrium calculations for Fe VIII and Fe IX.

ionization balance calculations but keeping the other parameters fixed (see Fig. 12). Figures 11 and 12 show:

- the importance of including Fe VIII in the calculation of the TRACE responses, in particular for the $195 \AA$ band;

- the large differences of the predicted TRACE 195/173 $\AA$ ratios when using different ion fractions;

- that the ratio curves are multi-valued, making it difficult to derive an unique temperature;

- that the temperature diagnostic has a large uncertainty at temperatures of $1 \mathrm{MK}$ or lower, typical of active region quiescent loops.

We note that the use of any filter ratio method can be very misleading whenever the plasma emission along the line of sight is not isothermal, a rather common case in the solar corona. It is also important to keep in mind that if the in-flight relative calibration between the filters differs from the values of the ground calibration, this would cause an offset in the predicted ratio values. Finally, there are various other effects that should be taken into account for a proper use of the filter ratio method. One is
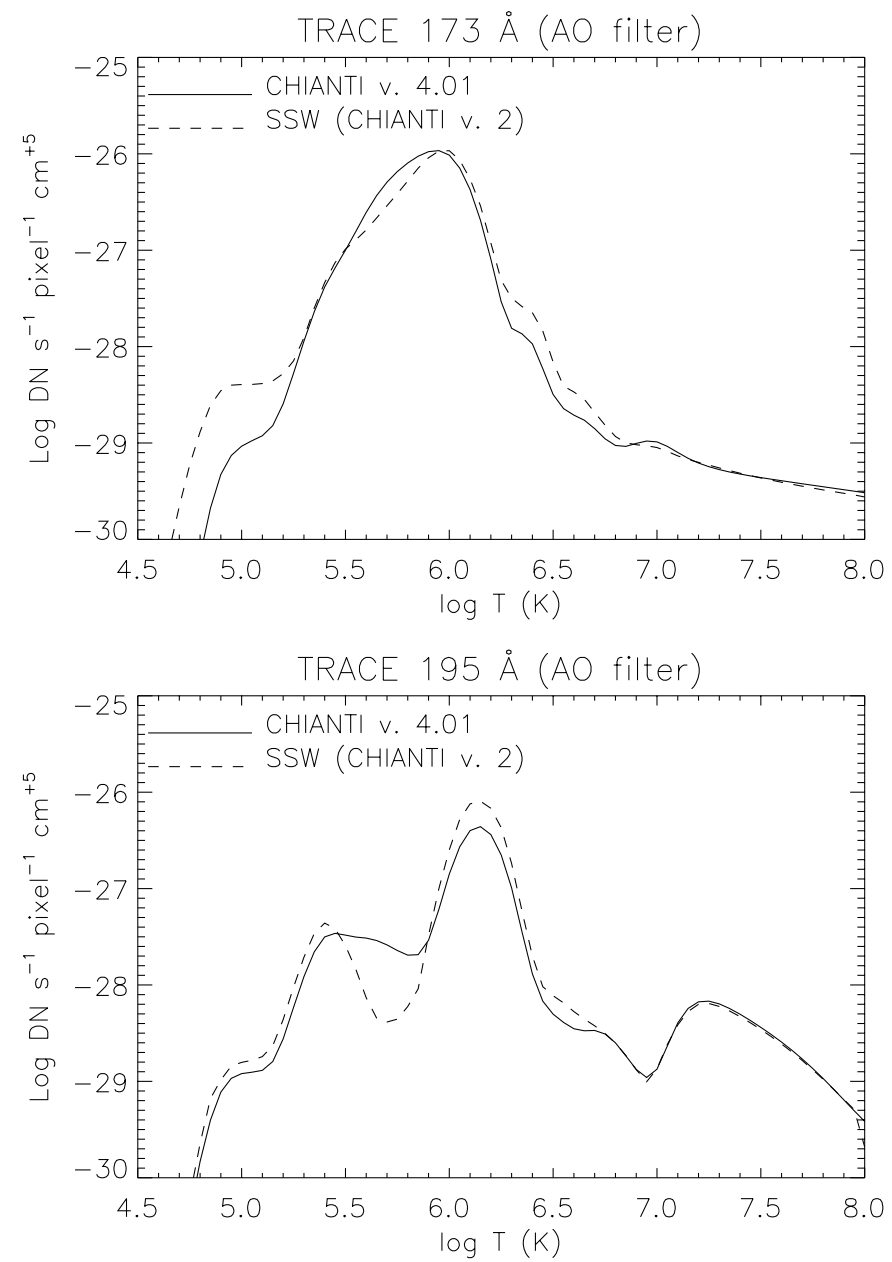

Fig. 11. The TRACE responses, calculated at constant emission measure by folding the CHIANTI V.4 emissivities with the TRACE effective areas, using a set of standard parameters. For comparison, the standard TRACE responses, available via Solarsoft (SSW) and calculated with CHIANTI V.2 are displayed.

related to the unknown element abundances. Another is related to uncertainties in the theoretical calculations of the emissivities of ions such as Fe IX, Fe X, Fe XII. A reliable assessment of the TRACE filter ratio method will only be possible when we have more accurate atomic calculations and after an in-flight calibration has been performed.

\subsection{The TRACE temperature measurements}

Having established the main characteristics of the filter ratio method and derived new TRACE response curves, we can now proceed to determine what temperatures could be derived from the TRACE data. We have already shown in Table 2 the TRACE count rates for the five loop leg areas of the loop and background. To extend these data to almost the half-length of the loop, we have selected an area along the loop and an adjacent one for the background (Fig. 13). The loop area was chosen as small as possible to reduce any background contamination, that is partly present in the five loop leg areas. 


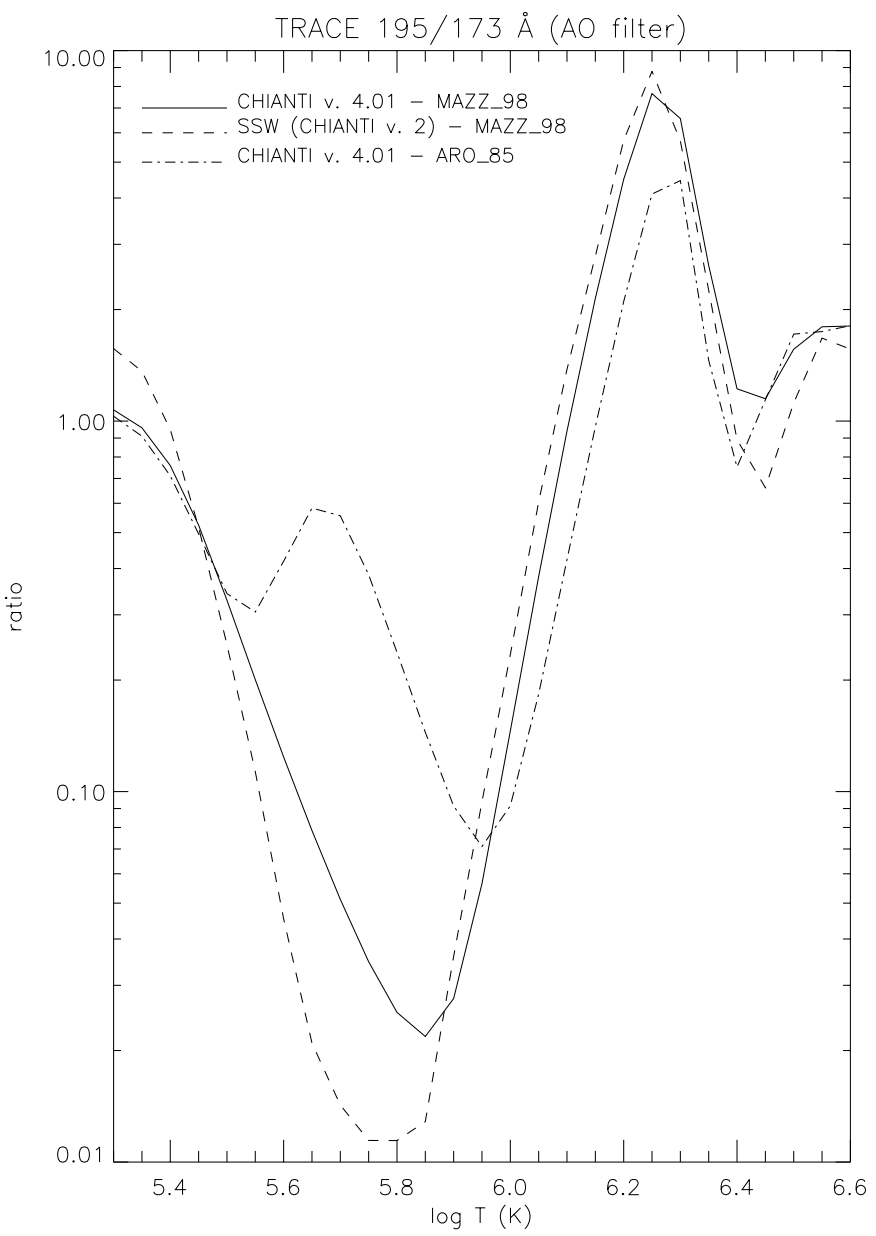

Fig. 12. The TRACE $195 / 173 \AA$ A ratio calculated using different ionization balance calculations (MAZZ_98: Mazzotta et al. 1998; ARO_85: Arnaud \& Rothenflug 1985). The standard ratio values available within Solarsoft (SSW) are also displayed for comparison. Note the large differences between the current SSW values and the new ones, the uncertainty associated with the use of different ionization balances and the multi-value character of the ratio.

Figure 14 shows the averaged count rates of the loop and the background regions, together with the 195/173 A ratio, calculated with and without background subtraction. Note that in the $110-140^{\prime \prime}$ region there was an interconnecting loop that contaminated the signal in both loop and background areas. With this in mind, we can see that:

1) the background intensities represent a significant fraction of the signal in the loop, all along its length, for both passbands;

2) for the $195 \AA$ band, the background signal is so high that it causes a very large uncertainty to be associated with the background-subtracted loop count rates;

3) the background-subtracted $195 / 173 \AA$ ratio values are in the range $0.1-0.6$, i.e. they fall in the region where the TRACE filter ratio method does not have a good temperature sensitivity and is most uncertain (see Fig. 12). If we assume that the ground calibration is applicable, we can see that the ratio values near the base of the loop are consistent with an increase of the temperature from 0.5 to $0.7 \mathrm{MK}$ in the first $15^{\prime \prime}$ (using Arnaud \& Rothenflug 1985), followed by a region (the loop leg) where

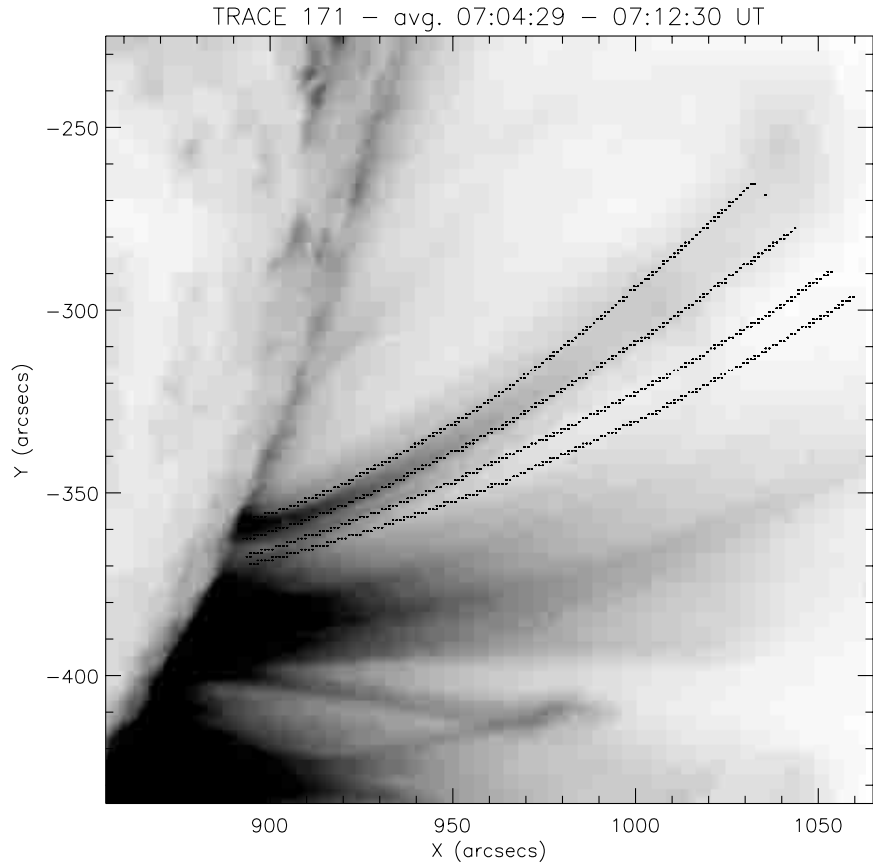

Fig. 13. The areas selected to obtain average TRACE count rates of the loop and background.

the temperature is about $1 \mathrm{MK}$, a result roughly consistent with those obtained with CDS. Toward the loop top, the ratio reaches a value of 0.4 , indicating that the loop does not reach temperatures higher than 1.1-1.2 MK (depending on which ionization balance is used). Again, this is is consistent with the maximum temperatures derived from the CDS for the other loops present in the dataset;

4) the ratio values without background subtraction are much higher (from 0.9 to 1.4) and would give incorrect temperatures ranging from values of $1.2-1.4 \mathrm{MK}$ at the loop leg (in stark contrast to the CDS results) toward values in the $1.3-1.5 \mathrm{MK}$ range close to the loop top.

Note that all the 195/173 $\AA$ ratio values for the four loops reported by Lenz et al. (1999) are in the range 0.9-1.4, exactly the same as those found here when the background is not subtracted. We have re-analysed loop b of Lenz (1999) et al. and can confirm that their temperatures were overestimated because the background emission was not subtracted. In fact, the derived temperatures were an average between the loop temperatures and those of the diffuse background emission. Obviously, also the emission measures (and hence the densities) were overestimated. We note that Aschwanden et al. (2000) do mention background subtraction, but still find temperatures above $1 \mathrm{MK}$ at the loop legs. This might be due to the fact that the loop extraction regions used (see their Fig. 3) still include some background, or to the fact that the background was contaminated. In fact, in off-limb observations it is very difficult to find suitable background regions, because of the multitude of loop emission that can be found along the line of sight.

Finally, we have performed various tests, which have shown that both the selection of the loop and background areas significantly affect the results. 

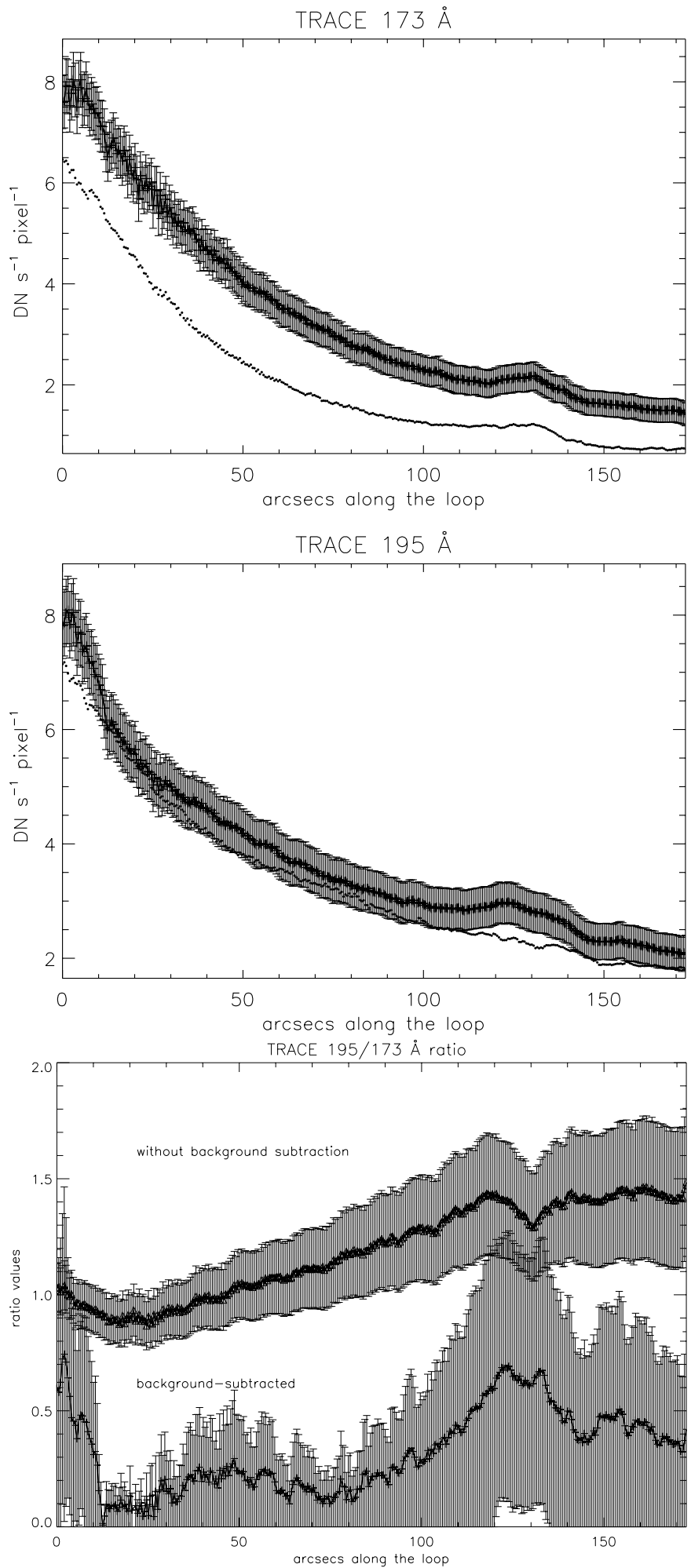

Fig. 14. Top two plots: the TRACE $173,195 \AA$ averaged count rates of the loop (crosses, with error bars) and background (circles) areas displayed in Fig. 13. Bottom plot: the TRACE 195/173 A ratios obtained with the above count rates. Note the large effect that background subtraction has. The ratios values in the 110-140" region are contaminated by the presence of a weak interconnecting loop.

\subsection{Forward modelling}

The best way to check the consistency of the derived physical values (in particular the density) is to carry out a form of forward modelling and predict the observed line intensities. Accurate forward modelling will only be possible once the TRACE bands have been calibrated in-flight and the atomic data improved. Here, we present a simple equilibrium model applied to the loop leg. We have assumed: 1) that the plasma is strictly isothermal along the line of sight; 2) the temperatures derived from the emission measure methods; 3 ) the coronal abundances of Feldman (1992); 4) a constant loop width of 7"; 5 ) a filling factor of 0.3 for the $0-8^{\prime \prime}$ region (points A, B), a linear increase up to 1.0 in the $8-25^{\prime \prime}$ interval, and 1.0 in the $25-60$ " region.

We have considered the TRACE $173 \AA$ band, and we fitted the TRACE profiles as in Fig. 5 for all points along the loop (assuming circular cross-section). We have taken the maximum values of the curves as representative of the count rates at the loop centre, and removed the same background as in Fig. 14. The peak values thus obtained correspond to the TRACE $173 \AA$ emission corresponding to a path length equal to the width of the loop. These values are shown in Fig. 15 (open squares), where we also show the values not background-subtracted (filled circles). Figure 15 also shows the observed CDS line intensities.

We have searched for a density profile that is both broadly consistent with the values measured directly from Mg VII and the CDS intensities. We have performed various tests by also allowing the temperatures to vary. We have found that, as expected, the observed intensities are most sensitive to variations in the temperature. This is due to the fact that both for the CDS emission lines and the TRACE $173 \AA$ band, the responses are strongly peaked in temperature. The intensities of course also depend on the square of the density.

The results, calculated with Arnaud \& Rothenflug (1985) are shown in Fig. 15 as solid lines. Those obtained with the Mazzotta et al. (1998) ionization balance are displayed with dashed lines. It is evident that the results for the CDS lines are not substantially dependent on the chosen ionization balance, while the same is not true for the TRACE $173 \AA$ band.

Given the fact that this is a simple model, the agreement between predicted and observed intensities is quite good, also considering all the uncertainties, the main one being background subtraction. The densities that reproduce the CDS line intensities decrease with height and are in broad agreement with the values obtained from the backgroundsubtracted Mg VII line ratios. Broad agreement with the absolute TRACE $173 \AA$ count rates is also found near the base of the loop, while at higher heights the $173 \AA$ count rates are under-estimated.

\section{Summary and conclusions}

We have presented simultaneous TRACE multi-filter and CDS observations of $1 \mathrm{MK}$ loops in a quiescent active region. The CDS observations allow a detailed study of the plasma properties of these loops, complementing the TRACE observations. Some important new results are presented here:

1) the loops are nearly isothermal along the line of sight, but show a temperature decrease toward their bases. 2) These loops are much cooler $(T=0.7-1 \mathrm{MK})$ than the surrounding 

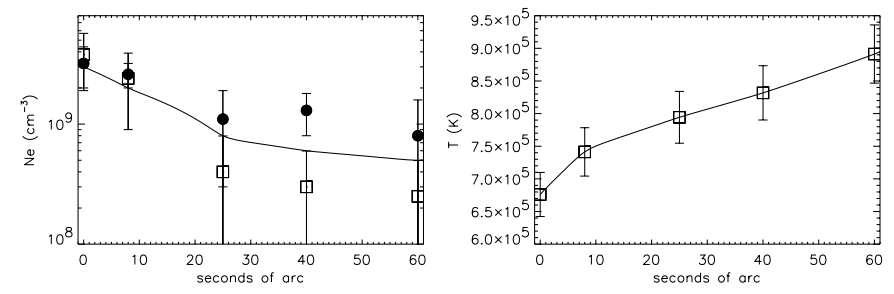

TRACE $173 \AA$

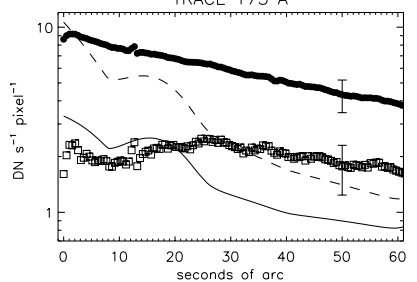

Ne VII $561.38+561.73(\AA)$

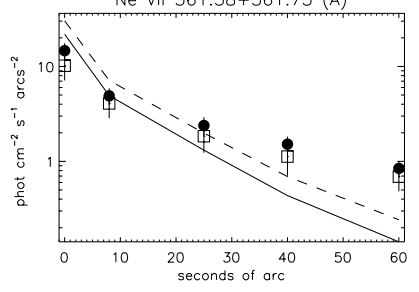

Si VIII $319.84(\AA \grave{A})$
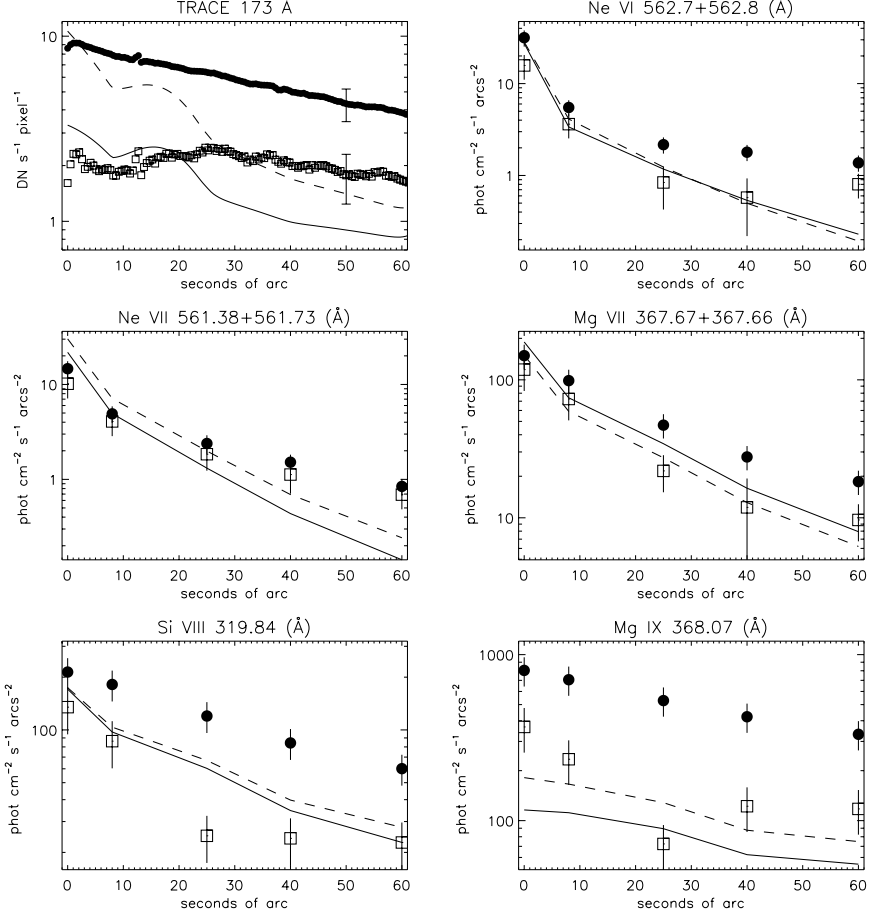

Mg VII $367.67+367.66(\AA)$
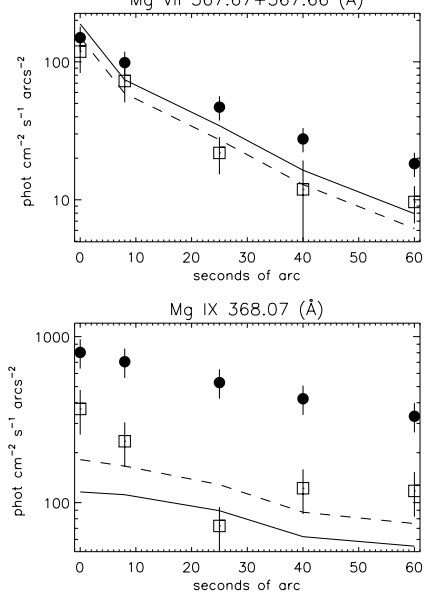

Fig. 15. From top to bottom: the assumed model densities and temperatures (solid lines), with the measured values (see Table 2) overplotted; the peak TRACE $173 \AA$ count rates with the predicted values; the observed and calculated intensities of some CDS lines along the leg of the loop. For both TRACE and CDS the loop values (filled circles) and the corresponding background-subtracted values (open squares) are plotted. The solid lines are the values calculated with the Arnaud \& Rothenflug (1985), while the dashed ones are calculated with the Mazzotta et al. (1998) ion balance.

diffuse high-lying active region emission $(T \simeq 2 \mathrm{MK})$, which is not isothermal along the line of sight. 3) We find severe limitations in the TRACE diagnostics, in particular in the filter ratio method applied to derive temperatures for the $1 \mathrm{MK}$ loops. The TRACE results previously obtained should be revised to take into account the uncertainties in the atomic data. 4) The diffuse background constitutes the bulk of the quiescent high-lying active region emission. Hence, any loop analysis (either spectroscopic or based on images) should take into account background subtraction. 5) Previous analyses of TRACE data have overestimated temperatures and densities because the background was not properly subtracted. 6) When the correct background subtraction is applied, the CDS and the TRACE observations become consistent. 7) The CDS observations are consistent with an FIP effect of about 4 (coronal abundances) and with filling factors close to unity. 8) The TRACE observations are consistent with loops having a constant density across their width.
Our results on the temperatures are at odds with the findings of most of the recent literature on the subject, where coronal loops were found to have an almost flat temperature distribution along their length. The fact that the $1 \mathrm{MK}$ loops are nearly isothermal along the line of sight is also different from the findings of Schmelz et al. (2001) and Martens et al. (2002), that found a flat DEM at some locations of a loop observed by CDS. However, it should be noted that the AR loop which they observed was of a very different type from those discussed here (because it was visible in lines formed in the $0.5-2 \mathrm{MK}$ temperature range).

Uncertainties are large, but we can rule out loop filling factors much lower than one, and we are therefore tempted to argue that the smallest threads seen by TRACE do not have many filamentary structures below the TRACE resolution $\left(0.5^{\prime \prime}\right.$, equivalent to $\left.365 \mathrm{~km}\right)$. Instruments such as Solar-B/EIS should be able to confirm this in the future.

We have presented some of the main characteristics of the diffuse background, showing how different it is from the emission of the $1 \mathrm{MK}$ quiescent loops. The diffuse background might well comprise a superposition of individual strands of loop emission below the TRACE resolution. Only instruments with a much higher spatial resolution might resolve this issue.

The results of the various analyses suggest that as a first approximation the loop plasma is in ionization equilibrium. However, some departures from equilibrium can occur, in particular if flows are present. These departures would affect the emissivities of the ions with longer timescales and would change the ion population (see Bradshaw \& Mason 2003). From the CDS observations we can rule out the presence of strong flows (say $50 \mathrm{~km} \mathrm{~s}^{-1}$ ) in the loops. However, moderate flows could be present. Indeed, recently Winebarger et al. (2002) reported measurements of $20 \mathrm{~km} \mathrm{~s}^{-1}$ flows at the base of some $1 \mathrm{MK}$ loops.

We have shown that coronal hole plume bases and legs of 1 MK loops have similar thermal characteristics. The number of similarities does not end here. Both plumes and these types of loops have simple geometries, are anchored in regions of high magnetic flux concentrations, and have periodical variations in intensities, interpreted as slow magnetoacustic waves (see, e.g., De Moortel et al. 2002, and references therein). One of the main differences is due to the fact that plumes do not show a significant FIP effect (Del Zanna et al. 2003).

Finally, a few concluding remarks. Our conclusions are based on a limited set of data and will need to be confirmed with further analyses. To our knowledge this is the first time that such a detailed comparison of CDS and TRACE data has been carried out for AR loops. It was extremely difficult to find a suitable dataset and loop. We have presented results based on one single loop, but we have also found similar characteristics in other loops in the same dataset. We have carried out preliminary analyses of other CDS and TRACE datasets which confirm our conclusions. We are therefore tempted to suggest that the characteristics described here are those of the "typical $1 \mathrm{MK}$ " loops. We are still searching for an ideal dataset in which an AR loop is observed along its full length, with the footpoints also clearly visible. The general properties of the $1 \mathrm{MK}$ loop footpoints in terms of densities, temperatures, 
elemental abundances, and relation to the underlying photospheric magnetic field are described in Del Zanna (2003), using on-disc SOHO and TRACE observations.

In this paper we have focused on the diagnostic methods used with CDS and TRACE and presented a few key new results. We have outlined the limitations of the techniques, assumptions and the atomic data. We have not attempted to relate these results to theoretical models. This is the subject of a separate study. Above all, our analysis clearly shows the importance of complementing imaging observations with spectroscopic data in order to accurately and unambiguously describe the characteristics of any solar feature.

Acknowledgements. We acknowledge support from PPARC. We thank J. Doyle for helpful comments on the manuscript and J. Raymond for providing ion balance calculations. The CDS observing sequence was designed by P. R. Young. We appreciate the useful discussions at the MEDOC workshop on "Coronal Loops" held in November 2002. SOHO is a project of international collaboration between ESA and NASA. We acknowledge the SURF and TRACE archives for providing data.

\section{References}

Arnaud, M., \& Raymond, J. 1992, ApJ, 398, 394

Arnaud, M., \& Rothenflug, R. 1985, A\&AS, 60, 425

Aschwanden, M. J. 2002, ApJ, 580, L79

Aschwanden, M. J., Newmark, J. S., Delaboudinière, J., et al. 1999, ApJ, 515, 842

Aschwanden, M. J., Nightingale, R. W., \& Alexander, D. 2000, ApJ, 541,1059

Bradshaw, S., \& Mason, H. E. 2003, A\&A, 401, 699

Bray, R. J. 1991, Plasma loops in the solar corona (Cambridge; New York: Cambridge University Press)

Brekke, P., Kjeldseth-Moe, O., \& Harrison, R. A. 1997, Sol. Phys., 175,511

Chae, J., Park, Y., Moon, Y., Wang, H., \& Yun, H. S. 2002, ApJ, 567, L159

Cheng, C.-C. 1980, ApJ, 238, 743

De Moortel, I., Ireland, J., Hood, A. W., \& Walsh, R. W. 2002, A\&A, 387, L13

Del Zanna, G. 1999, Ph.D. Thesis, Univ. of Central Lancashire, UK

Del Zanna, G. 2002, in The Radiometric Calibration of SOHO, ISSI Scientific Report SR-002, 283

Del Zanna, G., \& Bromage, B. J. I. 1999, Space Sci. Rev., 87, 169

Del Zanna, G., Bromage, B. J. I., Landi, E., \& Landini, M. 2001a, A\&A, 379, 708

Del Zanna, G., Bromage, B. J. I., \& Mason, H. E. 2001b, in Solar and Galactic Composition, AIP Conf. Proc., 598, 59

Del Zanna, G., Bromage, B. J. I., \& Mason, H. E. 2003, A\&A, 398, 743

Del Zanna, G., Gibson, S. E., Mason, H. E., Pike, C. D., \& Mandrini, C. H. 2002a, Adv. Space Res., 30, 551

Del Zanna, G., Landini, M., \& Mason, H. E. 2002b, A\&A, 385, 968
Del Zanna, G. 2003, A\&A, 406, L5

Delaboudiniere, J.-P., Artzner, G. E., Brunaud, J., et al. 1995, Sol. Phys., 162, 291

Dere, K. P. 1982, Sol. Phys., 75, 189

Doyle, J. G., Mason, H. E., \& Vernazza, J. E. 1985, A\&A, 150, 69

Feldman, U. 1992, Phys. Scr, 46, 202

Fludra, A., Brekke, P., Harrison, R. A., et al. 1997, Sol. Phys., 175, 487

Fludra, A., Del Zanna, G., Alexander, D., \& Bromage, B. J. I. 1999, J. Geophys. Res., 104, 9709

Fludra, A., \& Schmelz, J. T. 1999, A\&A, 348, 286

Gabriel, A. H., \& Jordan, C. 1975, MNRAS, 173, 397

Gibson, S. E., Fletcher, L., Del Zanna, G., et al. 2002, ApJ, 574, 1021

Handy, B. N., Acton, L. W., \& Kankelborg, C. C. et al. 1999, Sol. Phys., 187, 229

Harrison, R. A., Sawyer, E. C., Carter, M. K., et al. 1995, Sol. Phys., 162,233

Haugan, S. V. H. 1997, CDS software note, 47

Kjeldseth-Moe, O., \& Brekke, P. 1998, Sol. Phys., 182, 73

Landi, E., Landini, M., Dere, K. P., Young, P. R., \& Mason, H. E. 1999, A\&AS, 135, 339

Lang, J., Thompson, W. T., Pike, C. D., Kent, B. J., \& Foley, C. R. 2002, in The Radiometric Calibration of SOHO, ISSI Scientific Report SR-002, 105

Lenz, D. D., Deluca, E. E., Golub, L., Rosner, R., \& Bookbinder, J. A. 1999, ApJ, 517, L155

Martens, P. C. H., Cirtain, J. W., \& Schmelz, J. T. 2002, ApJ, 577, L115

Mason, H. E., Landi, E., Pike, C. D., \& Young, P. R. 1999, Sol. Phys., 189, 129

Mason, H. E., \& Monsignori Fossi, B. C. M. 1994, A\&ARv, 6, 123

Mason, H. E., Young, P. R., Pike, C. D., et al. 1997, Sol. Phys., 170, 143

Mazzotta, P., Mazzitelli, G., Colafrancesco, S., \& Vittorio, N. 1998, A\&AS, 133, 403

Monsignori Fossi, B. C., \& Landini, M. 1991, Adv. Space Res., 11, 281

Neupert, W. M., Newmark, J., Delaboudinière, J.-P., et al. 1998, Sol. Phys., 183, 305

Orrall, F. Q., ed. 1981, Solar active regions: A monograph from SKYLAB Solar Workshop III

Pike, C. D., \& Mason, H. E. 2002, Sol. Phys., 206, 359

Raymond, J. C., \& Foukal, P. 1982, ApJ, 253, 323

Raymond, J. C., Mazur, J. E., Allegrini, F., et al. 2001, in Solar and Galactic Composition, AIP Conf. Proc., 598, 49

Reale, F., \& Peres, G. 2000, ApJ, 528, L45

Schmelz, J. T. 2002, ApJ, 578, L161

Schmelz, J. T., Scopes, R. T., Cirtain, J. W., Winter, H. D., \& Allen, J. D. 2001, ApJ, 556, 896

Schrijver, C. J., Title, A. M., Berger, T. E., et al. 1999, Sol. Phys., 187, 261

Serio, S., Peres, G., Vaiana, G. S., Golub, L., \& Rosner, R. 1981, ApJ, 243, 288

Testa, P., Peres, G., Reale, F., \& Orlando, S. 2002, ApJ, 580, 1159

Winebarger, A. R., Warren, H., van Ballegooijen, A., DeLuca, E. E., \& Golub, L. 2002, ApJ, 567, L89

Young, P. R., Del Zanna, G., Landi, E., et al. 2003, ApJS, 144, 135 